\title{
Properties of centered random walks on locally compact groups and Lie groups
}

Nick Dungey

\begin{abstract}
The basic aim of this paper is to study asymptotic properties of the convolution powers $K^{(n)}=K * K * \cdots * K$ of a possibly nonsymmetric probability density $K$ on a locally compact, compactly generated group $G$. If $K$ is centered, we show that the Markov operator $T$ associated with $K$ is analytic in $L^{p}(G)$ for $1<p<\infty$, and establish Davies-Gaffney estimates in $L^{2}$ for the iterated operators $T^{n}$. These results enable us to obtain various Gaussian bounds on $K^{(n)}$. In particular, when $G$ is a Lie group we recover and extend some estimates of Alexopoulos and of Varopoulos for convolution powers of centered densities and for the heat kernels of centered sublaplacians. Finally, in case $G$ is amenable, we discover that the properties of analyticity or Davies-Gaffney estimates hold only if $K$ is centered.
\end{abstract}

\section{Introduction and statement of results}

The general aim of this paper is to study some properties of non-symmetric random walks on a locally compact group $G$. We consider a probability density $K$ on $G$ and the random walk on $G$ governed by $K$. Under certain assumptions on $K$, we prove time regularity estimates and Gaussian offdiagonal estimates for this walk. While such estimates are well known for symmetric random walks on groups or for symmetric continuous time diffusions on Lie groups (see, for example, [22, 38, 5] and references therein), our results make no symmetry assumption.

It is remarkable that our results apply on arbitrary locally compact, compactly generated groups without any special assumptions on the group

2000 Mathematics Subject Classification: 60B15, 60G50, 22E30, 22D05, 35B40.

Keywords: Locally compact group, Lie group, amenable group, random walk, probability density, heat kernel, Gaussian estimates, convolution powers. 
structure (though it turns out that the results are most interesting for amenable groups). However, in much of the paper we deal with the case where $G$ is a connected Lie group, since the arguments in this case are technically easier and of independent interest. The extension to arbitrary compactly generated groups is accomplished in the last part of the paper.

In the Lie group case, we recover and extend some results of Alexopou$\operatorname{los}[1,4,2]$ and Varoupoulos [37] for non-symmetric random walks and heat kernels on Lie groups. (See also [26] for the special case of nilpotent Lie groups.) Note that the analyses of $[4,2,37]$ depend on probabilistic methods, while our approach is completely different and is functional-analytic rather than probabilistic. Indeed, our approach essentially generalizes [16] where the case of a discrete group $G$ was studied. The advantage of our functional-analytic approach, in comparison to methods of $[4,2,37]$, is that it does not rely on detailed knowledge of the structure theory or geometry of $G$, so that it applies when such structure theory is not available (for example, on discrete groups).

To explain our results more precisely, recall (cf. $[10,5])$ that a bounded linear operator $S \in \mathcal{L}(X)$, where $X$ is a complex Banach space, is said to be analytic if there exists a $c>0$ such that

$$
\left\|(I-S) S^{n}\right\| \leq c n^{-1}
$$

for all $n \in \mathbb{N}=\{1,2,3, \ldots\}$. This notion is a discrete time analogue of the usual notion of analyticity for a continuous time semigroup $\left(e^{t A}\right)_{t \geq 0}$ which corresponds to an estimate $\left\|e^{t A}\right\| \leq c t^{-1}, t>0$. We establish the fundamental result that the Markov operator $T$, associated with the density $K$ on $G$, is analytic in $L^{2}(G)$ whenever $K$ is centered (the definition of "centered" is given below). This theorem provides a large and interesting class of examples of non-self-adjoint analytic operators.

Our second fundamental theorem gives "Davies-Gaffney estimates", that is, $L^{2}$ off-diagonal estimates, for the iterated operators $T^{n}$ when $K$ is centered. This enables us to deduce Gaussian estimates for the $n$-th convolution powers $K^{(n)}$ of $K$. Similar Gaussian estimates occur for symmetric random walks and heat kernels in, for example, [34, 8, 22, 38, 28], or for non-symmetric walks on Lie groups in $[4,2,37]$. But for non-symmetric walks on general groups, our Gaussian estimates are apparently new and not deducible from previously known results.

When $G$ is amenable we discover that the above properties, that is, analyticity of $T$ or the Davies-Gaffney estimates, hold if and only if the density $K$ is centered. 
As already mentioned, our results and methods for locally compact groups are based on those of [16] for discrete groups. The present paper is technically more difficult than [16], since the topology of $G$ leads to problems which do not occur in the discrete case.

To state our results precisely we fix some ideas and notation (for relevant background material, see [38]). In general, $G$ will denote a compactly generated, locally compact group and $d g$ will be a left invariant Haar measure on $G$. Let $d \widehat{g}=d\left(g^{-1}\right)=\Delta\left(g^{-1}\right) d g$ denote right Haar measure on $G$, where $\Delta: G \rightarrow(0, \infty)$ is the modular function.

Let $K$ be a probability density on $G$ with respect to $d g$, that is, $K: G \rightarrow$ $[0, \infty)$ is a Borel measurable function with $\int_{G} d g K(g)=1$.

The density $K$ determines a random walk on $G$, whose distribution after $n$ steps is given by the $n$-th convolution power $K^{(n)}:=K * K * \cdots * K$, $n \in \mathbb{N}$. Here, in general the convolution of two functions $f_{1}, f_{2}$ on $G$ is given by

$$
\left(f_{1} * f_{2}\right)(g)=\int_{G} d h f_{1}(h) f_{2}\left(h^{-1} g\right)
$$

for all $g \in G$. The Markov operator $T$ associated with $K$ is defined by

$$
T f:=K * f
$$

for any $f \in L^{p}:=L^{p}(G ; d g), 1 \leq p \leq \infty$. Then $\|T\|_{p \rightarrow p} \leq 1$ for all $p \in[1, \infty]$, where $\|\cdot\|_{p \rightarrow q}$ denotes the norm of a bounded linear operator from $L^{p}$ to $L^{q}$. Note that $T^{n} f=K^{(n)} * f$ for all $n \in \mathbb{N}$.

Let $\left(f_{1}, f_{2}\right):=\int_{G} d g f_{1} \overline{f_{2}}$ denote the inner product of complex-valued functions in $L^{2}=L^{2}(G ; d g)$. It is easy to see that $T$ is self-adjoint (that is, $\left.\left(T f_{1}, f_{2}\right)=\left(f_{1}, T f_{2}\right), f_{1}, f_{2} \in L^{2}\right)$ if and only if $K$ is symmetric in the sense that $K(g)=\Delta\left(g^{-1}\right) K\left(g^{-1}\right), g \in G$, but we shall not assume symmetry.

Let $L=L_{G}$ be the left regular representation of $G$ so that $(L(g) f)(h)=$ $f\left(g^{-1} h\right)$ for a function $f: G \rightarrow \mathbb{C}$ and $g, h \in G$. Define the difference operators $\partial_{g}:=L(g)-I$ for $g \in G$. By definition, the "discrete Laplacian" corresponding to $K$ is the operator

$$
I-T=I-\int_{G} d g K(g) L(g)=-\int_{G} d g K(g) \partial_{g} .
$$

Since $G$ is compactly generated, we may define a distance on $G$ in the following standard way. For the rest of the paper, we fix a relatively compact, open neighborhood $U$ of the identity $e$ of $G$ which is symmetric (that is, $U=U^{-1}$ ) and generates $G$. Then $G=\bigcup_{n=1}^{\infty} U^{n}$ where $U^{n}:=$ $\left\{g_{1} g_{2} \cdots g_{n}: g_{1}, \ldots, g_{n} \in U\right\}$, and we define $\rho=\rho_{U}: G \rightarrow \mathbb{N}$ by

$$
\rho(g):=\inf \left\{n \in \mathbb{N}: g \in U^{n}\right\}, \quad g \in G .
$$


One has $\rho(g)=\rho\left(g^{-1}\right)$ and $\rho(g h) \leq \rho(g)+\rho(h)$ for all $g, h \in G$. It is well known that the behaviour of $\rho$ is essentially independent of the choice of $U$ (precisely, if $\rho^{\prime}$ is the distance associated with another such neighborhood $U^{\prime}$, then $c^{-1} \rho \leq \rho^{\prime} \leq c \rho$ for some constant $c>1$ ).

Recall that $G$ is said to have polynomial volume growth of order $D$ $(D \geq 0)$ if one has an estimate

$$
c^{-1} n^{D} \leq d g\left(U^{n}\right) \leq c n^{D}
$$

for all $n \in \mathbb{N}$. Such groups are necessarily unimodular. Alternatively, if there exists $a>0$ with $d g\left(U^{n}\right) \geq a e^{a n}, n \in \mathbb{N}$, then $G$ is said to have exponential volume growth. Every connected Lie group is either of polynomial growth or exponential growth (see [20]), but there exist examples of finitely generated discrete groups whose growth is intermediate, that is, neither polynomial nor exponential ([19]).

Let $L_{l o c}^{p}$ be the space of functions on $G$ which are locally in $L^{p}$. We will often make use of the " $L^{p}$ gradient" $\Gamma_{p}(f)$ of a function $f \in L_{l o c}^{p}(G)$, defined by

$$
\Gamma_{p}(f):=\sup _{u \in U}\left\|\partial_{u} f\right\|_{p} \in[0,+\infty]
$$

To study "off-diagonal" properties of the random walk, and in particular to obtain Gaussian estimates on $K^{(n)}$, our main technical tool will be the perturbations $T_{\lambda}=e^{\lambda \psi} T e^{-\lambda \psi}, \lambda \in \mathbb{R}$, of the Markov operator $T$. Here, $\psi: G \rightarrow \mathbb{R}$ is a suitable function and the operator $T_{\lambda}$ is formally defined by

$$
T_{\lambda} f=e^{\lambda \psi} T\left(e^{-\lambda \psi} f\right), \quad f \in L^{p} .
$$

The $\left\{T_{\lambda}\right\}_{\lambda \in \mathbb{R}}$ may be called "Davies perturbations" of $T$, in analogy with the standard Davies perturbation of semigroups generated by differential operators (for the latter see $[12,13]$ ).

The class of $\psi$ we consider is the class $\mathcal{E}=\mathcal{E}_{G, U}$ of all locally bounded Borel measurable functions $\psi: G \rightarrow \mathbb{R}$ which satisfy

$$
\Gamma_{\infty}(\psi)=\sup _{u \in U}\left\|\partial_{u} f\right\|_{\infty} \leq 1
$$

Note that $\psi$ itself is not assumed to be bounded. It is clear from the triangle inequality $\rho(g h) \leq \rho(g)+\rho(h), g, h \in G$, that $\rho \in \mathcal{E}$.

In Subsections 1.1 to 1.4 below, we state our main results. The organization of the rest of the paper is described in Subsection 1.5. 


\subsection{Densities on Lie groups}

To state our main results for Lie groups, in this subsection we shall assume that $G$ is a connected Lie group. In this case we usually make the following assumptions on the density $K$.

(i) There exists a neighborhood $W$ of the identity $e$ of $G$ such that

$$
\inf \{K(g): g \in W\}>0 .
$$

(ii) For every $k>0$, one has

$$
\int_{G} d g K(g) e^{k \rho(g)}<\infty .
$$

Assumption (ii) requires that $K$ decreases sufficiently fast at infinity. For example, let us say that $K$ is a sub-Gaussian density if $K$ satisfies Assumption (i) and an upper Gaussian estimate of the form

$$
K(g) \leq c e^{-b \rho(g)^{2}}
$$

for some constants $c, b>0$ and all $g \in G$. Then a sub-Gaussian density satisfies Assumption (ii), by the well known fact that $\int_{G} d g e^{-\delta \rho(g)^{2}}$ is finite for any $\delta>0$. Note that any compactly supported, bounded density which satisfies (i) is sub-Gaussian.

The concept of centeredness for densities on a Lie group is defined as follows (cf. [4,37]). Let $G_{0}$ be the closure in $G$ of the commutator subgroup $[G, G]$, and consider the canonical homomorphism $\pi_{0}: G \rightarrow G / G_{0}$. Observe that $G / G_{0}$ is a connected abelian Lie group and therefore it can be written in the form

$$
G / G_{0} \cong \mathbb{R}^{q} \times \mathbb{T}^{r}
$$

for some $q, r \in\{0,1,2, \ldots\}$, where $\mathbb{T}:=\mathbb{R} / \mathbb{Z}$. Define $G_{1}:=\pi_{0}^{-1}\left(\{0\} \times \mathbb{T}^{r}\right)$. Then $G_{1}$ is a closed normal subgroup of $G$, with $[G, G] \subseteq G_{1}$ and $G_{1} / G_{0} \cong$ $\mathbb{T}^{r}, G / G_{1} \cong \mathbb{R}^{q}$. Consider the homomorphism $\pi_{1}: G \rightarrow G / G_{1} \cong \mathbb{R}^{q}$ and let $\pi_{1}^{(i)}: G \rightarrow \mathbb{R}$ be the $i$-th component of $\pi_{1}$, for $i \in\{1, \ldots, q\}$. We say that the density $K$ is centered if the first order moments of the projection of $K$ onto $\mathbb{R}^{q}$ vanish, that is, if

$$
\int_{G} d g K(g) \pi_{1}^{(i)}(g)=0
$$

for all $i \in\{1, \ldots, q\}$. 
It is not difficult to show that $K$ is centered if and only if $K * \eta=\eta$ for all continuous homomorphisms $\eta: G \rightarrow \mathbb{R}$ (observe that any such homomorphism vanishes on $G_{1}$, and must therefore be a linear combination of the homomorphisms $\pi_{1}^{(i)}$.)

The following lemma (whose proof is just as in [4, Section 1.11]) shows that a general density is conjugate, via a multiplicative function, to a centered density.

Lemma 1.1 Let $K$ be a non-centered density on $G$ satisfying Assumptions (i) and (ii) above. There exist a centered density $K^{\prime}$, a smooth multiplicative function $\chi: G \rightarrow(0, \infty)$ (that is, $\chi(g h)=\chi(g) \chi(h)$ for all $g, h \in G$ ) and a constant $\delta \in(0,1)$ such that

$$
K(g)=\delta \chi(g) K^{\prime}(g)
$$

for all $g \in G$. Setting $T^{\prime} f:=K^{\prime} * f$, we have the relations $K^{(n)}(g)=$ $\delta^{n} \chi(g)\left(K^{\prime}\right)^{(n)}(g)$ and $T^{n} f=\delta^{n} \chi\left(T^{\prime}\right)^{n}\left(\chi^{-1} f\right)$ for all $n \in \mathbb{N}, g \in G$ and $f \in L^{p}, 1 \leq p \leq \infty$.

In this sense, the study of general densities reduces to the study of centered densities, and in what follows we concentrate mainly on the centered case.

We now state our first main result.

Theorem 1.2 Let $K$ be a centered density on $G$ satisfying Assumptions (i) and (ii) above. Then $T$ is analytic in $L^{2}$, that is, there exists $c>0$ with $\left\|(I-T) T^{n}\right\|_{2 \rightarrow 2} \leq c n^{-1}$ for all $n \in \mathbb{N}$.

The following result is crucial for the proof of Theorem 1.2.

Theorem 1.3 Let $K$ be a centered density on $G$ satisfying Assumptions (i) and (ii). Then there exists a $c>0$ such that $|((I-T) f, f)| \leq c \Gamma_{2}(f)^{2}$ for all $f \in L^{2}$.

A theorem of Blunck [6] states that if an operator $S \in \mathcal{L}\left(L^{p_{1}}\right) \cap \mathcal{L}\left(L^{p_{2}}\right)$ is analytic in $L^{p_{1}}$ and power-bounded in both $L^{p_{1}}$ and $L^{p_{2}}$ (power-bounded means that $\left.\sup _{n \in \mathbb{N}}\left\|S^{n}\right\|<\infty\right)$, then $S$ is analytic in $L^{q}$ for any $q$ strictly between $p_{1}$ and $p_{2}$. Since $\left\|T^{n}\right\|_{p \rightarrow p} \leq 1$ for all $p \in[1, \infty]$, this theorem implies the following corollary of Theorem 1.2.

Corollary 1.4 Under the hypotheses of Theorem 1.2, then $T$ is analytic in $L^{p}$ for each $p \in(1, \infty)$. 
Our next result is the fundamental $L^{2}$ off-diagonal estimate for centered sub-Gaussian densities.

Theorem 1.5 Let $K$ be a sub-Gaussian density on $G$ which is centered. There exists a $\omega>0$ such that, for all $\psi \in \mathcal{E}$, setting $T_{\lambda}=e^{\lambda \psi} T e^{-\lambda \psi}$ we have

$$
\left\|T_{\lambda}^{n}\right\|_{2 \rightarrow 2} \leq e^{\omega \lambda^{2} n}
$$

for all $n \in \mathbb{N}$ and $\lambda \in \mathbb{R}$.

From Theorem 1.5 one can deduce the following estimates. Define the distance between two subsets $E, F$ of $G$ by $d(E, F)=\inf \left\{\rho\left(g h^{-1}\right): g \in\right.$ $E, h \in F\}$. Let $\chi_{E}$ denote the characteristic function of $E \subseteq G$ (thus $\chi_{E}(g)=1$ or 0 according as $g \in E$ or $g \notin E$ ), and also denote by $\chi_{E}$ the operator of pointwise multiplication $f \mapsto \chi_{E} f$.

Theorem 1.6 Let $K$ be a sub-Gaussian density which is centered. Given any $p \in(1, \infty)$, there exist positive constants $c=c(p), b=b(p)$ depending on $p$ such that

$$
\left\|\chi_{E} T^{n} \chi_{F}\right\|_{p \rightarrow p} \leq c e^{-b d(E, F)^{2} / n}
$$

for all $n \in \mathbb{N}$ and all non-empty Borel measurable sets $E, F \subseteq G$.

We refer to the estimates of Theorems 1.5 and 1.6 as $L^{2}$ (or $L^{p}$ ) offdiagonal estimates or Davies-Gaffney estimates. (The cases $p=1$ or $p=\infty$ are discussed in the Remarks at the end of this subsection.) Analogous estimates are well known for symmetric random walks (see for example [22, 9]) or for heat semigroups on manifolds (for example, [13, 18]).

The next theorem shows that when $G$ is amenable, the $L^{2}$ estimates of Theorems 1.2, 1.5 or 1.6 characterize centered densities. (An analogous result on discrete groups was established in [16].)

Theorem 1.7 Suppose $G$ is amenable and $K$ is a sub-Gaussian density. Then the following conditions (I) to (VI) are equivalent.

(I) $K$ is centered.

(II) $T$ is analytic in $L^{2}$.

(III) There exists $c>0$ such that

$$
|((I-T) f, f)| \leq c \Gamma_{2}(f)^{2}
$$

for all $f \in L^{2}$.

(IV) For each $\psi \in \mathcal{E}$, there exist $c, \omega>0$ such that setting $T_{\lambda}=e^{\lambda \psi} T e^{-\lambda \psi}$ we have

$$
\left\|T_{\lambda}^{n}\right\|_{2 \rightarrow 2} \leq c e^{\omega \lambda^{2} n}
$$

for all $n \in \mathbb{N}$ and $\lambda \in[-1,1]$. 
(V) For each $\psi \in \mathcal{E}$, setting $T_{\lambda}=e^{\lambda \psi} T e^{-\lambda \psi}$ one has

$$
\left\|T_{\lambda}\right\|_{2 \rightarrow 2}=1+O\left(\lambda^{2}\right)
$$

for all $\lambda \in[-1,1]$.

(VI) There exist $c, b>0$ such that

$$
\left\|\chi_{E} T^{n} \chi_{F}\right\|_{2 \rightarrow 2} \leq c e^{-b d(E, F)^{2} / n}
$$

for all $n \in \mathbb{N}$ and non-empty Borel measurable sets $E, F \subseteq G$.

In stating Theorem 1.7 we used the standard notation $O\left(\lambda^{k}\right), \lambda \in J$, to denote a function $s=s(\lambda)$ satisfying an estimate $|s(\lambda)| \leq c\left|\lambda^{k}\right|$ for all $\lambda$ in an interval $J \subseteq \mathbb{R}$.

Remark. It is well known that, for a density $K$ on $G$ satisfying Assumption (i), one has $\|T\|_{2 \rightarrow 2}<1$ if and only if $G$ is not amenable (see, for example, [31, pp. 140-142]). When $G$ is not amenable, using $\|T\|_{2 \rightarrow 2}<1$ it is rather easy to see that Conditions (II) through (VI) of Theorem 1.7 hold even for non-centered densities. (For example, Conditions (IV) and (V) follow from $\|T\|_{2 \rightarrow 2}<1$ and the perturbation estimate of Lemma 4.1 below.) Therefore, our results in $L^{2}$ are mainly of interest for amenable groups.

We proceed to state a number of results which are essentially derivable from the fundamental Theorems 1.2 and 1.5. The following result was proved on amenable Lie groups in [37], by quite different methods (and for a slightly smaller class of densities $K$ ).

Theorem 1.8 ([37]) Let $K$ be a sub-Gaussian density which is centered. There exist $c, b>0$ such that

$$
K^{(n)}(g) \leq c \Delta(g)^{-1 / 2} e^{-b \rho(g)^{2} / n}
$$

for all $n \in \mathbb{N}$ and $g \in G$.

We have the following analyticity and spatial regularity estimates for the perturbed operators $T_{\lambda}$.

Theorem 1.9 Let $K$ be sub-Gaussian and centered. Set $T_{\lambda}:=e^{\lambda \psi} T e^{-\lambda \psi}$ where $\lambda \in \mathbb{R}$ and $\psi \in \mathcal{E}$. There exist constants $c, \omega>0$ independent of $\psi$, such that

$$
\begin{gathered}
\left\|\left(I-T_{\lambda}\right) T_{\lambda}^{n}\right\|_{2 \rightarrow 2}=\left\|e^{\lambda \psi}(I-T) T^{n} e^{-\lambda \psi}\right\|_{2 \rightarrow 2} \leq c n^{-1} e^{\omega \lambda^{2} n}, \\
\left\|e^{\lambda \psi} \partial_{h} T^{n} e^{-\lambda \psi}\right\|_{2 \rightarrow 2}+\left\|\partial_{h} T_{\lambda}^{n}\right\|_{2 \rightarrow 2} \leq c \rho(h) n^{-1 / 2} e^{\omega \lambda^{2} n},
\end{gathered}
$$

for all $n \in \mathbb{N}, \lambda \in \mathbb{R}$, and $h \in G$ satisfying $\rho(h) \leq n^{1 / 2}$. 
In case $G$ has polynomial growth, Theorems 1.5 and 1.9 , together with the arguments of [14], lead to a new proof of the following precise Gaussian estimates. These estimates were established in [4] for compactly supported densities, by quite different arguments involving homogenization theory.

Theorem 1.10 Suppose $G$ is a Lie group of polynomial volume growth of order D. Let $K$ be sub-Gaussian and centered. Then there are $c, b>0$ such that

$$
K^{(n)}(g)+n^{1 / 2}\left|\left(\partial_{h} K^{(n)}\right)(g)\right|+n\left|K^{(n)}(g)-K^{(n+1)}(g)\right| \leq c n^{-D / 2} e^{-b \rho(g)^{2} / n}
$$

for all $n \in \mathbb{N}, g \in G$ and $h \in U$.

Remarks. (a) If the Lie group $G$ is amenable and if $K$ is centered, then the $L^{p}$ off-diagonal estimate (1.5) also holds in the cases $p=1$ and $p=\infty$. This fact is essentially contained in the results of [37].

Indeed, the probabilistic estimate of [37, inequality (0.9)] on an amenable Lie group implies that

$$
\int_{\rho(g) \geq r} d g K^{(n)}(g) \leq c e^{-b r^{2} / n}
$$

for all $n \in \mathbb{N}$ and $r \geq 1$. (A version of (1.6) for symmetric heat kernels was also obtained in [21].) It is straightforward to show that (1.6) is equivalent to the case $p=1$ ( or $p=\infty$ ) of (1.5).

(b) As already mentioned in [37], estimate (1.6) fails on non-amenable groups, and also fails on certain amenable discrete groups of exponential growth. Consequently, the case $p=1$ of (1.5) fails on such groups.

This means that the $L^{1}$ case of (1.5) is essentially stronger than the $L^{2}$ case, for we shall see (see Subsection 1.4) that the $L^{2}$ case holds on arbitrary compactly generated groups.

Note finally that (1.6) holds when $G$ is a Lie group of polynomial growth, by a standard integration of the Gaussian estimate of Theorem 1.10.

(c) When $G$ has polynomial volume growth, the hypothesis in Theorem 1.2 that Assumption (ii) holds can be replaced by a weaker, polynomial decay condition on $K$. For details, see the Remarks at the end of Section 3.

\subsection{Time inhomogeneous walks on Lie groups}

Let $G$ be a connected Lie group. In this subsection, we observe that some of the above results extend to time inhomogeneous random walks on $G$. That is, given some sequence $\left(K_{n}\right)_{n=1}^{\infty}$ of densities on $G$, one may consider a random walk whose distribution after $n$ steps is given by the convolution

$$
K_{n} * K_{n-1} * \cdots * K_{1}, \quad n \in \mathbb{N} .
$$


We have the following estimates (which generalize Theorems 1.5 and 1.8) provided that the $K_{n}$ satisfy uniform assumptions.

Theorem 1.11 Let $\left(K_{n}\right)_{n=1}^{\infty}$ be a sequence of centered densities on $G$. Assume that (i) there exists a neighborhood $W$ of e with $\inf \left\{K_{n}(g): g \in W, n \in\right.$ $\mathbb{N}\}>0$, and (ii) there are $c, b>0$ with $K_{n}(g) \leq c e^{-b \rho(g)^{2}}$ for all $g \in G$, $n \in \mathbb{N}$. Define $T_{n} f:=K_{n} * f, f \in L^{p}$. Then there exist constants $\omega, c^{\prime}, b^{\prime}>0$ such that

$$
\left\|e^{\lambda \psi} T_{m+n} \cdots T_{m+1} e^{-\lambda \psi}\right\|_{2 \rightarrow 2} \leq e^{\omega \lambda^{2} n}
$$

for all $m \in \mathbb{N}_{0}=\{0,1,2, \ldots\}, n \in \mathbb{N}, \lambda \in \mathbb{R}, \psi \in \mathcal{E}$, and

$$
\left(K_{m+n} * \cdots * K_{m+1}\right)(g) \leq c^{\prime} \Delta(g)^{-1 / 2} e^{-b^{\prime} \rho(g)^{2} / n}
$$

for all $m \in \mathbb{N}_{0}, n \in \mathbb{N}$ and $g \in G$.

The first estimate of Theorem 1.10 extends to time inhomogeneous walks as follows.

Theorem 1.12 Suppose that the densities $\left(K_{n}\right)_{n=1}^{\infty}$ satisfy the hypotheses of Theorem 1.11, and that $G$ has polynomial volume growth of order $D$. Then

$$
\left(K_{m+n} * \cdots * K_{m+1}\right)(g) \leq c n^{-D / 2} e^{-b \rho(g)^{2} / n}
$$

for all $m \in \mathbb{N}_{0}$ and $n \in \mathbb{N}$.

In contrast to Theorem 1.10, Theorem 1.12 does not appear to be obtainable from the homogenization methods of [4].

Given Theorem 1.12, then the arguments of [37] probably allow one to extend (1.6) to time inhomogeneous walks on amenable Lie groups. We will not, however, give the details.

In analogy with Theorem 1.2, one can ask whether estimates of type

$$
\left\|\left(I-T_{n+1}\right) T_{n} T_{n-1} \cdots T_{1}\right\|_{2 \rightarrow 2} \leq c n^{-1}, \quad n \in \mathbb{N}
$$

are valid, where $T_{j}$ denotes the Markov operator associated with $K_{j}$. But the proof of Theorem 1.2 does not yield such estimates and we do not know when they are true.

We do, though, have the following statement that a family of centered densities satisfying uniform assumptions is uniformly analytic. This result will be usefully applied to study the semigroup generated by a sublaplacian (see Subsection 1.3 below). 
Theorem 1.13 Let $\left(K_{(\alpha)}\right)_{\alpha \in A}$ be a family of centered densities on $G$, indexed by a set $A$. Assume that (i) there exists a neighborhood $W$ of e with $\inf \left\{K_{(\alpha)}(g): g \in W, \alpha \in A\right\}>0$, and (ii) $\sup _{\alpha \in A} \int_{G} d g K_{(\alpha)}(g) e^{k \rho(g)}<\infty$ for each $k>0$. Set $T_{(\alpha)} f=K_{(\alpha)} * f, f \in L^{p}$. Then for each $p \in(1, \infty)$, there exists a $c=c(p)>0$ with

$$
\left\|\left(I-T_{(\alpha)}\right) T_{(\alpha)}^{n}\right\|_{p \rightarrow p} \leq c n^{-1}
$$

for all $n \in \mathbb{N}$ and $\alpha \in A$.

\subsection{Sublaplacians with drift}

In this subsection, $G$ will again denote a connected Lie group. An important application of the theory of Subsection 1.1 above is the study of the semigroup and heat kernel associated with a sublaplacian operator on $G$.

To state some known and some new results for sublaplacians, let $\mathfrak{g}$ be the Lie algebra of $G$, consisting of all right invariant vector fields on $G$. Fix elements $A_{0}, A_{1}, \ldots, A_{d^{\prime}} \in \mathfrak{g}$ such that $A_{1}, \ldots, A_{d^{\prime}}$ algebraically generate the Lie algebra $\mathfrak{g}$, and consider the subelliptic sublaplacian

$$
H=-\sum_{i=1}^{d^{\prime}} A_{i}^{2}+A_{0}
$$

with drift term $A_{0}$. It is well known (see for example [32, Section IV.4]) that $H$ generates a contraction semigroup $\left(e^{-t H}\right)_{t \geq 0}$ in $L^{p}, 1 \leq p \leq \infty$. We denote by $K_{t}: G \rightarrow(0, \infty)$ the corresponding heat kernel which satisfies $e^{-t H} f=K_{t} * f$ and $K_{t} * K_{s}=K_{t+s}$ for all $t, s>0$ and $f \in L^{p}$.

For each fixed $t>0, K_{t}$ is a sub-Gaussian density on $G$ and in fact also satisfies a lower Gaussian bound (see [35, Appendix A.4]).

One says that $H$ is centered if $A_{0} \in \mathfrak{g}_{1}$, where $\mathfrak{g}_{1} \subseteq \mathfrak{g}$ denotes the Lie algebra of $G_{1}$. It is straightforward to show that $H$ is centered if and only if $K_{t}$ is centered for all $t>0$.

Centered sublaplacians have been studied by various methods in $[2,37$, $26,15]$. In this paper, we shall prove the following large time regularity result for the semigroup $e^{-t H}$, essentially as a consequence of Theorem 1.13.

Theorem 1.14 Let $H$ be a centered sublaplacian on $G$. For each $p \in$ $(1, \infty)$, there exists $c=c(p)>0$ such that

$$
\left\|H e^{-t H}\right\|_{p \rightarrow p} \leq c t^{-1}
$$

for all $t \geq 1$. 
Using Theorems 1.10 and 1.14, we will give a new proof of the following Gaussian estimates due to Alexopoulos [2].

Theorem 1.15 ([2]). Let $G$ have polynomial growth of order $D$ and let $H$ be a centered sublaplacian on $G$. Given any right invariant vector field $X$ on $G$, one has estimates

$$
K_{t}(g)+t^{1 / 2}\left|X K_{t}(g)\right|+t\left|\frac{d}{d t} K_{t}(g)\right| \leq c t^{-D / 2} e^{-b \rho(g)^{2} / t}
$$

for all $t \geq 1$ and $g \in G$.

Alternative proofs of the first estimate $K_{t}(g) \leq c t^{-D / 2} e^{-b \rho(g)^{2} / t}, t \geq 1$, of Theorem 1.15 were given in [15] and (in the case of nilpotent groups) in [26].

Note that when $G$ has polynomial growth, a standard integration of the Gaussian estimate on $(d / d t) K_{t}=-H K_{t}$ of Theorem 1.15 implies the estimate of Theorem 1.14, for any $p \in[1, \infty]$. But when $G$ has exponential growth, the estimate of Theorem 1.14 appears to be new.

\subsection{Locally compact groups}

In this subsection, we allow $G$ to be any locally compact, compactly generated group. It is remarkable that the main results of Subsections 1.1 and 1.2 extend to this general situation.

For simplicity, we make the following assumption of compact support on the density $K$.

(i)' $K$ is compactly supported, bounded, and $\inf \{K(g): g \in W\}>0$, where $W$ is some relatively compact, open and symmetric neighborhood of the identity $e$ of $G$ which generates $G$.

The notion of centeredness is extended to this general situation as follows. Set $G_{0}=\overline{[G, G]}$ and consider the projection $\pi_{0}: G \rightarrow G / G_{0}$. Since $G / G_{0}$ is a compactly generated locally compact abelian group, by a standard structure theorem (see [23, Theorem II.9.8]) it can be written as a direct product $G / G_{0} \cong \mathbb{R}^{q} \times \mathbb{Z}^{r} \times M$ where $q, r \in\{0,1,2, \ldots\}$ and $M$ is a compact abelian group. Set $G_{1}=\pi_{0}^{-1}(\{0\} \times M)$. Then $G_{1}$ is a closed normal subgroup of $G$ with $[G, G] \subseteq G_{1}$, and $G_{1} / G_{0} \cong M$ is compact. Consider the homomorphism $\pi_{1}: G \rightarrow G / G_{1} \cong \mathbb{R}^{q} \times \mathbb{Z}^{r}$ with components $\pi_{1}^{(i)}: G \rightarrow \mathbb{R}, i \in\{1, \ldots, q\}$, and $\pi_{1}^{(i)}: G \rightarrow \mathbb{Z}, i \in\{q+1, \ldots, q+r\}$. We say that $K$ is centered if (1.4) holds for each $i \in\{1, \ldots, q+r\}$.

Just as in the Lie group case, one sees that $K$ is centered if and only if $K * \eta=\eta$ (or, equivalently, $(I-T) \eta=0)$ for every continuous homomorphism $\eta: G \rightarrow \mathbb{R}$ 
If $K$ is a non-centered density satisfying (i)', then there exists a centered density $K^{\prime}$ such that $K=\delta \chi K^{\prime}$ where $\delta \in(0,1)$ is a constant and $\chi: G \rightarrow$ $(0, \infty)$ is a continuous multiplicative function. The proof of this fact is similar to the proof of Lemma 1.1 (cf. $[4,3]$ ) and is left to the reader.

Concerning analyticity of $T$, we have the following extension of Theorems 1.2 and 1.3 .

Theorem 1.16 Let $G$ be a locally compact, compactly generated group and let $K$ be a centered density on $G$ satisfying Assumption (i)'. Then there exists $c>0$ such that

$$
\left|\left((I-T) f_{1}, f_{2}\right)\right| \leq c \Gamma_{2}\left(f_{1}\right) \Gamma_{2}\left(f_{2}\right)
$$

for all $f_{1}, f_{2} \in L^{2}$. Moreover, $T$ is analytic in $L^{p}$ for each $p \in(1, \infty)$.

We also have off-diagonal estimates generalizing Theorems 1.5, 1.6 and 1.8, as follows.

Theorem 1.17 Let $G$ be a locally compact, compactly generated group, and let $K$ be a centered density on $G$ satisfying Assumption (i)'. Then the following statements hold.

(I) There is $c>0$ such that

$$
\left|\left((I-T) f_{1}, f_{2}\right)\right| \leq c \Gamma_{\infty}\left(f_{1}\right) \Gamma_{1}\left(f_{2}\right)
$$

for all $f_{1} \in L^{\infty}$ and $f_{2} \in L^{1}$.

(II) There is $\omega>0$ such that

$$
\left\|e^{\lambda \psi} T^{n} e^{-\lambda \psi}\right\|_{2 \rightarrow 2} \leq e^{\omega \lambda^{2} n}
$$

for all $n \in \mathbb{N}, \lambda \in \mathbb{R}, \psi \in \mathcal{E}$. For each $p \in(1, \infty)$ there exist $c=c(p)$, $b=b(p)>0$ such that

$$
\left\|\chi_{E} T^{n} \chi_{F}\right\|_{p \rightarrow p} \leq c e^{-b d(E, F)^{2} / n}
$$

for all $n \in \mathbb{N}$ and all Borel sets $E, F \subseteq G$, where $d(E, F)=\inf \left\{\rho\left(g h^{-1}\right): g \in\right.$ $E, h \in F\}$.

(III) There are $c, b>0$ such that

$$
K^{(n)}(g) \leq c \Delta(g)^{-1 / 2} e^{-b \rho(g)^{2} / n}
$$

for all $n \in \mathbb{N}$ and $g \in G$. 
Moreover, the estimates of Theorem 1.9 hold when $K$ is centered. As a consequence, we can generalize Theorem 1.10 for any locally compact group of polynomial growth.

Theorem 1.18 Suppose that $G$ is a locally compact, compactly generated group of polynomial volume growth of order $D$. Let $K$ be a centered density on $G$ satisfying Assumption (i)'. Then there are $c, b>0$ such that

$$
K^{(n)}(g)+n^{1 / 2}\left|\left(\partial_{h} K^{(n)}\right)(g)\right|+n\left|K^{(n)}(g)-K^{(n+1)}(g)\right| \leq c n^{-D / 2} e^{-b \rho(g)^{2} / n}
$$

for all $n \in \mathbb{N}, g \in G$ and $h \in U$.

For amenable groups we have the following extension of Theorem 1.7.

Theorem 1.19 Let $G$ be an amenable locally compact, compactly generated group and $K$ a density on $G$ satisfying Assumption (i)'. Then the Conditions (I) through (VI) in Theorem 1.7 are all equivalent.

If $G$ is non-amenable then the situation is as follows (compare the Lie group case in Subsection 1.1): for any, possibly non-centered, density $K$ satisfying Assumption (i)', one has $\|T\|_{2 \rightarrow 2}<1$ and Conditions (II) through (VI) in Theorem 1.7 all hold.

Remark. Let us mention some further $L^{\infty}$ estimates and Gaussian estimates for $K^{(n)}$. Observe that if $K$ is centered and one has a uniform upper bound of the form

$$
\left\|\Delta^{1 / 2} K^{(n)}\right\|_{\infty} \leq \gamma(n)
$$

for all $n \in \mathbb{N}$ and some function $\gamma: \mathbb{N} \rightarrow(0, \infty)$, then interpolation with the bound of Theorem 1.17, part (III), yields that

$$
\Delta(g)^{1 / 2} K^{(n)}(g) \leq \gamma(n)^{1-\varepsilon} c^{\varepsilon} e^{-b \varepsilon \rho(g)^{2} / n}
$$

for any $\varepsilon \in(0,1), g \in G$ and $n \in \mathbb{N}$. For example, if (1.11) holds with $\gamma(n)=c_{1} e^{-c_{2} n^{\beta}}$ where $\beta \in(0,1]$, then we obtain

$$
K^{(n)}(g) \leq c_{3} \Delta(g)^{-1 / 2} e^{-c_{4} n^{\beta}} e^{-c_{4} \rho(g)^{2} / n}
$$

for some constants $c_{3}, c_{4}>0$.

For example, if $G$ is unimodular and of exponential volume growth then (1.11) holds with $\gamma(n)=c_{1} e^{-c_{2} n^{1 / 3}}$, by a well known theorem (see [38, Chapter VII]). Therefore, in this case an estimate of type

$$
K^{(n)}(g) \leq c e^{-b n^{1 / 3}} e^{-b \rho(g)^{2} / n}
$$

holds for all $n \in \mathbb{N}, g \in G$, when $K$ is centered. This appears to be a new result for non-symmetric densities. 
Finally, for time inhomogeneous random walks on $G$ we record the following extension of Theorem 1.17.

Theorem 1.20 Let $G$ be a locally compact, compactly generated group and let $\left(K_{n}\right)_{n=1}^{\infty}$ be a sequence of compactly supported, centered densities on $G$. Define $T_{n} f:=K_{n} * f, f \in L^{p}$. Suppose there exist $\varepsilon>0$ and two relatively compact, symmetric generating neighborhoods $W, W^{\prime}$ of the identity e such that $W \subseteq W^{\prime}, W$ is open, and

$$
\varepsilon \chi_{W} \leq K_{n} \leq \varepsilon^{-1} \chi_{W^{\prime}}
$$

for all $n \in \mathbb{N}$. Then there exist $\omega, c, b>0$ such that

$$
\left\|e^{\lambda \psi} T_{m+n} \cdots T_{m+1} e^{-\lambda \psi}\right\|_{2 \rightarrow 2} \leq e^{\omega \lambda^{2} n}
$$

for all $m \in \mathbb{N}_{0}, n \in \mathbb{N}, \lambda \in \mathbb{R}, \psi \in \mathcal{E}$, and

$$
\left(K_{m+n} * \cdots * K_{m+1}\right)(g) \leq c \Delta(g)^{-1 / 2} e^{-b \rho(g)^{2} / n}
$$

for all $m \in \mathbb{N}_{0}, n \in \mathbb{N}$ and $g \in G$.

\subsection{Organization of the paper}

An outline of the paper is as follows.

In Section 2 we begin the proof of Theorem 1.2 for a Lie group; the proof is completed in Section 3 with the proof of Theorem 1.3. The underlying idea of the proof of Theorem 1.3 is that, roughly speaking, if $K$ is centered then $I-T$ is a second-order difference operator without first-order terms, that is, it is a generalized linear combination of operators $\partial_{g_{1}} \cdots \partial_{g_{k}}$ where $g_{1}, \ldots, g_{k} \in G$ and $k \geq 2$ (see [16] for the analogous assertion on discrete groups). To develop this idea precisely on Lie groups, however, requires some effort.

In Section 4 we establish Theorem 1.5. For this proof we need a certain variation of the estimate of Theorem 1.3 (see (3.15) below).

The ideas and results of Sections 2, 3 and 4 are fundamental for this article, and are used repeatedly in later sections.

Next, sections 5 and 6 contain the proofs of Theorems 1.6 and 1.7 respectively. The proof of Theorem 1.6 is based on an equivalence between two different forms of $L^{p}$ off-diagonal estimates, and shows that Theorems 1.6 and 1.5 are essentially equivalent.

Section 7 describes the proofs of Theorems 1.8 to 1.13 , utilizing results in Sections 2 to 4.

In Section 8, we consider sublaplacians on the Lie group $G$, and prove Theorems 1.14 and 1.15 . 
Finally, in Sections 9 and 10 we consider general locally compact compactly generated groups and derive the results stated in Subsection 1.4. The proofs are based on similar ideas as in the Lie group case, but significant difficulties occur and we rely on some deep structure theorems for locally compact groups (cf. [27]).

In general, $c, c^{\prime}, b$ and so on will denote positive constants whose value may change from line to line when convenient.

\section{Proof of Theorem 1.2}

In this section, unless otherwise stated $G$ will be a connected Lie group. Note, however, that the arguments of this section do not use Lie theory and extend (with only minor changes) to locally compact, compactly generated groups.

To prove Theorem 1.2 we will apply the following general characterization of analytic operators due to Nevanlinna (see [29, Theorem 4.5.4], [30, Theorem 2.1], and $[5,6])$. Let $\mathbb{D}=\{\lambda \in \mathbb{C}:|\lambda|<1\}$ be the open unit disk.

Theorem 2.1 Let $X$ be a complex Banach space and let $S \in \mathcal{L}(X)$. The following three conditions are equivalent.

(I) $S$ is power-bounded (that is, $\sup \left\{\left\|S^{n}\right\|: n \in \mathbb{N}\right\}<\infty$ ) and analytic.

(II) $\left(e^{-t(I-S)}\right)_{t \geq 0}$ is a bounded analytic semigroup in $X$, and the spectrum of $S$ is contained in $\mathbb{D} \cup\{1\}$.

(III) There exists a $c>0$ such that $\left\|(\lambda I-S)^{-1}\right\| \leq c|\lambda-1|^{-1}$ for all $\lambda \in \mathbb{C}$ with $|\lambda|>1$.

To prove Theorem 1.2 we will verify Condition (II) of Theorem 2.1 when $S=T$ (where $T f:=K * f$ ) and $X=L^{2}=L^{2}(G ; d g)$. The following general proposition, and Assumption (i), yield the desired condition on the spectrum of $T$. Denote by $\sigma_{p}(S)$ the spectrum of an operator $S \in \mathcal{L}\left(L^{p}\right)$.

Proposition 2.2 Let $G$ be a locally compact group with left Haar measure $d g$, let $P: G \rightarrow[0, \infty)$ be a probability density on $G$, and define the operator $S$ by $S f=P * f, f \in L^{2}:=L^{2}(G ; d g)$. Suppose there exists a neighborhood $W$ of the identity of $G$ such that $\inf \{P(g): g \in W\}>0$. Then $\sigma_{2}(S) \subseteq \mathbb{D} \cup\{1\}$

The proof of Proposition 2.2 uses the following Hilbert space result. For any $\lambda \in \mathbb{C}, A \subseteq \mathbb{C}$, let us write $\operatorname{dist}(\lambda, A):=\inf \{|\lambda-a|: a \in A\}$.

Lemma 2.3 For any $S \in \mathcal{L}\left(L^{2}\right)$, the spectrum $\sigma_{2}(S)$ is contained in the closure $\overline{\Theta(S)}$ of the set $\Theta(S):=\left\{(S f, f): f \in L^{2},\|f\|_{2}=1\right\} \subseteq \mathbb{C}$. Moreover, for each $\lambda \in \mathbb{C} \backslash \overline{\Theta(S)}$, setting $d_{\lambda}:=\operatorname{dist}(\lambda, \overline{\Theta(S)})=\operatorname{dist}(\lambda, \Theta(S))>0$ one has $\left\|(\lambda I-S)^{-1}\right\|_{2 \rightarrow 2} \leq d_{\lambda}^{-1}$. 
The first statement of the lemma is standard (see [24, Corollary V.3.3]). The second statement then follows by observing that $\operatorname{dist}(\lambda, \Theta(S)) \leq \mid \lambda-$ $(S f, f) \mid \leq\|(\lambda I-S) f\|_{2}$ for $\|f\|_{2}=1$.

Proof of Proposition 2.2. Let $P$ and $W$ be as in the hypothesis, and choose a compact neighborhood $V$ of $e$ such that $V=V^{-1}$ and $V V \subseteq W$. Let $\Delta: G \rightarrow(0, \infty)$ be the modular function of $G$, so that $d \widehat{g}:=\Delta\left(g^{-1}\right) d g$ is right Haar measure on $G$. Set

$$
P_{1}:=\varepsilon\left(\Delta^{-1} \chi_{V}\right) * \chi_{V}
$$

where $\chi_{V}$ is the characteristic function of $V$ and $\varepsilon>0$ is a constant chosen small enough so that $P_{1} \leq P$. Define $S_{1} f:=P_{1} * f$ for all $f \in L^{2}$. It is easy to check that

$$
\left(S_{1} f, f\right)=\varepsilon\left(\chi_{V} * f, \chi_{V} * f\right) \geq 0,
$$

so that $S_{1}$ is non-negative self-adjoint in $L^{2}$. Put $\delta=\int_{G} d g P_{1}(g)$. Because $0 \leq P_{1} \leq P$, we have $0<\delta \leq 1$ and

$$
\left\|S_{1}\right\|_{2 \rightarrow 2} \leq \delta, \quad\left\|S-S_{1}\right\|_{2 \rightarrow 2} \leq \int_{G} d g\left(P-P_{1}\right)(g)=1-\delta .
$$

Let $\|f\|_{2}=1$. Since $\left(S_{1} f, f\right) \in[0, \delta]$, then $(S f, f)=\left(S_{1} f, f\right)+\left(\left(S-S_{1}\right) f, f\right)$ is an element of the set

$$
\Lambda_{\delta}:=\{\lambda \in \mathbb{C}: \operatorname{dist}(\lambda,[0, \delta]) \leq 1-\delta\} .
$$

By Lemma 2.3 and since $\delta \in(0,1]$, we conclude that $\sigma_{2}(S) \subseteq \overline{\Lambda_{\delta}}=\Lambda_{\delta} \subseteq$ $\mathbb{D} \cup\{1\}$.

By Theorem 2.1 and Proposition 2.2, the proof of Theorem 1.2 is reduced to showing that, when $K$ is centered, $e^{-t(I-T)}$ is a bounded analytic semigroup in $L^{2}$. To show this, by a well known semigroup result (see [24, Theorem IX.1.24]) it suffices to obtain a sectorial estimate of form

$$
|((I-T) f, f)| \leq c \operatorname{Re}((I-T) f, f)
$$

for all $f \in L^{2}$. We will begin the proof of $(2.2)$ in this section and will complete the proof in Section 3.

We will need the identities

$$
\partial_{g_{1} \cdots g_{n}}=\partial_{g_{1}}+L\left(g_{1}\right) \partial_{g_{2}}+\cdots+L\left(g_{1} \cdots g_{n-1}\right) \partial_{g_{n}}
$$

and

$$
\partial_{g_{1} \cdots g_{n}}=\partial_{g_{1}}+\cdots+\partial_{g_{n}}+\sum_{k=2}^{n} \sum_{i_{1}, \ldots, i_{k}} \partial_{g_{i_{1}}} \ldots \partial_{g_{i_{k}}}
$$

for all $n \in \mathbb{N}$ and $g_{1}, \ldots, g_{n} \in G$. In the second identity, the inner sum is over all integers $i_{1}, \ldots, i_{k}$ with $1 \leq i_{1}<i_{2}<\cdots<i_{k} \leq n$. 
604 N. Dungey

The next lemma records basic facts about $\Gamma_{p}$ defined by (1.2).

Lemma 2.4 One has

$$
\left\|\partial_{g} f\right\|_{p} \leq \rho(g) \Gamma_{p}(f)
$$

and

$$
\Gamma_{p}(L(g) f) \leq 3 \rho(g) \Gamma_{p}(f)
$$

for all $g \in G$ and $f \in L^{p}$. Given any relatively compact, Borel measurable neighborhood $V$ of $e$ in $G$, there exists a $c=c(V)>1$ such that

$$
c^{-1} \Gamma_{2}(f)^{2} \leq \int_{V} d h\left\|\partial_{h} f\right\|_{2}^{2} \leq c \Gamma_{2}(f)^{2}
$$

for all $f \in L^{2}$.

Proof. Inequality (2.5) follows easily by writing $g=g_{1} g_{2} \cdots g_{n}$ with $g_{j} \in U$, $n=\rho(g)$, and applying $(2.3)$.

To prove (2.6), note that

$$
\partial_{u} L(g) f=-\partial_{g} f+L(u) \partial_{g} f+\partial_{u} f
$$

for $u \in U, g \in G$. Therefore by (2.5),

$$
\left\|\partial_{u} L(g) f\right\|_{p} \leq 2\left\|\partial_{g} f\right\|_{p}+\left\|\partial_{u} f\right\|_{p} \leq(2 \rho(g)+1) \Gamma_{p}(f) \leq 3 \rho(g) \Gamma_{p}(f)
$$

for all $u \in U$ and $g \in G$, which implies (2.6).

In the final statement of the lemma, the upper bound on $\int_{V} d h\left\|\partial_{h} f\right\|_{2}^{2}$ follows easily from (2.5). The lower bound follows as in the proof of $[38$, Proposition VII.3.2] and we refer there for details.

Remark. The statements of Lemma 2.4 actually hold on any locally compact compactly generated group $G$, provided that $V$ is a relatively compact, open neighborhood of $e$ which generates $G$.

The first step in the proof of (2.2) is the following observation.

Lemma 2.5 There exists a $c>1$ such that

$$
c^{-1} \Gamma_{2}(f)^{2} \leq \operatorname{Re}((I-T) f, f) \leq c \Gamma_{2}(f)^{2}
$$

for all $f \in L^{2}$. 
Proof. Let $T^{*}$ be the $L^{2}$-adjoint of $T$ and consider the self-adjoint operator $\widehat{T}:=2^{-1}\left(T+T^{*}\right)$. For $f \in L^{2}$, note that

$$
\operatorname{Re}((I-T) f, f)=2^{-1}((I-T) f, f)+2^{-1}(f,(I-T) f)=((I-\widehat{T}) f, f)
$$

and that $\widehat{T} f=\widehat{K} * f$, where $\widehat{K}$ is the probability density

$$
\widehat{K}(g):=2^{-1}\left(K(g)+\Delta\left(g^{-1}\right) K\left(g^{-1}\right)\right), \quad g \in G .
$$

Using $d\left(g^{-1}\right)=\Delta\left(g^{-1}\right) d g$ and the symmetry $\widehat{K}(g)=\Delta\left(g^{-1}\right) \widehat{K}\left(g^{-1}\right)$, one checks that

$$
\begin{aligned}
2^{-1} \int_{G} d g \widehat{K}(g) & \partial_{g^{-1}} \partial_{g}=2^{-1} \int_{G} d g \widehat{K}(g)\left(2 I-L(g)-L\left(g^{-1}\right)\right) \\
& =I-2^{-1} \int_{G} d g \widehat{K}(g) L(g)-2^{-1} \int_{G} d\left(g^{-1}\right) \widehat{K}\left(g^{-1}\right) L(g) \\
& =I-\int_{G} d g \widehat{K}(g) L(g)=I-\widehat{T}
\end{aligned}
$$

Because $\partial_{g^{-1}}$ has adjoint $\partial_{g}$, then

$$
\begin{aligned}
((I-\widehat{T}) f, f) & =2^{-1} \int_{G} d g \widehat{K}(g)\left\|\partial_{g} f\right\|_{2}^{2} \\
& \leq 2^{-1} \int_{G} d g \widehat{K}(g) \rho(g)^{2} \Gamma_{2}(f)^{2} \\
& \leq c \Gamma_{2}(f)^{2}
\end{aligned}
$$

for all $f \in L^{2}$, since $\int_{G} d g \widehat{K}(g) \rho(g)^{2}<\infty$ by Assumption (ii).

Next, by Assumption (i) there exists a compact neighborhood $V^{\prime}$ of $e$ such that $\inf \left\{\widehat{K}(g): g \in V^{\prime}\right\}>0$. We obtain an estimate

$$
((I-\widehat{T}) f, f)=2^{-1} \int_{G} d g \widehat{K}(g)\left\|\partial_{g} f\right\|_{2}^{2} \geq c \int_{V^{\prime}} d g\left\|\partial_{g} f\right\|_{2}^{2} \geq c^{\prime} \Gamma_{2}(f)^{2},
$$

where the last step follows by the last statement of Lemma 2.4. The lemma follows.

Remark. The proof of Lemma 2.5 does not require that $K$ be centered: the lemma is valid for any density satisfying Assumptions (i) and (ii).

In Section 3, we will prove Theorem 1.3.

Combining Theorem 1.3 and Lemma 2.5 gives an estimate of form (2.2). Then $\left(e^{-t(I-T)}\right)_{t \geq 0}$ is a bounded analytic semigroup in $L^{2}$, and Theorem 1.2 follows. 


\section{Proof of Theorem 1.3}

Let $G$ be a connected Lie group with Lie algebra $\mathfrak{g}$. In this section we prove Theorem 1.3, which will complete the proof of Theorem 1.2.

A key element of the proof is to establish estimates of type

$$
\mid\left(\partial_{g} f_{1}, f_{2}\right) \leq c(g) \Gamma_{2}\left(f_{1}\right) \Gamma_{2}\left(f_{2}\right)
$$

for all $f_{1}, f_{2} \in L^{2}$, where $g$ is an element of the subgroup $G_{1}$ of $G$ and $c(g)>0$ is a constant which may depend on $g$. The idea behind the proof of such estimates is that when $g \in G_{1}$ then $\partial_{g}$ is a "second-order" difference operator in the sense that it is a linear combination of operators $\partial_{g_{1}} \cdots \partial_{g_{k}}$ where $k \geq 2, g_{1}, \ldots, g_{k} \in G$. To make this idea precise, we shall apply some structure theory of Lie groups. (Later, by analogous but more complicated arguments we shall obtain (3.1) on any locally compact compactly generated group; see Proposition 9.4.)

Recall that $[G, G]$ consists of all finite products of the commutators $\left[g_{1}, g_{2}\right]:=g_{1} g_{2} g_{1}^{-1} g_{2}^{-1}, g_{1}, g_{2} \in G$. It is well known (see [33, Theorem 3.18.7]) that $[G, G]$ is a connected, possibly non-closed, Lie subgroup of the connected Lie group $G$, and has Lie algebra $[\mathfrak{g}, \mathfrak{g}]$. The groups $G_{0}=\overline{[G, G]}$ and $G_{1}$, defined in Subsection 1.1, are closed, connected Lie subgroups of $G$. (Connectedness of $G_{1}$ follows from the connectedness of $G_{0}$ and of $G_{1} / G_{0} \cong \mathbb{T}^{r}$.) Denote by $\mathfrak{g}_{1}$ the Lie algebra of $G_{1}$.

Lemma 3.1 There exist $m \in \mathbb{N}$ and a compact symmetric neighborhood $U^{\prime}$ of e in $G$ such that, setting

$$
U^{\prime \prime}:=\left\{\left[g_{1}, g_{2}\right]: g_{1}, g_{2} \in U^{\prime}\right\}
$$

then the set $\left(U^{\prime \prime}\right)^{m}:=\left\{u_{1} \cdots u_{m}: u_{j} \in U^{\prime \prime}\right\}$ is a neighborhood of the identity for the Lie group $[G, G]$.

Proof. Let $m \in \mathbb{N}$ and consider the smooth mapping $\Psi: G^{2 m} \rightarrow[G, G]$ defined by

$$
\Psi\left(g_{1}, h_{1}, g_{2}, h_{2}, \ldots, g_{m}, h_{m}\right):=\left[g_{1}, h_{1}\right] \cdots\left[g_{m}, h_{m}\right]
$$

for all $g_{j}, h_{j} \in G$. The argument of [33, p.242] shows that if $m$ is chosen large enough, then the differential

$$
\left.\Psi_{*}\right|_{p}: T_{p}\left(G^{2 m}\right) \rightarrow T_{e}([G, G])
$$

is surjective at some point $p=\left(a_{1}, e, a_{2}, e, \ldots, a_{m}, e\right) \in G^{2 m}$, where $a_{1}, \ldots, a_{m}$ $\in G$. The lemma then follows by taking any compact symmetric neighborhood $U^{\prime}$ of $e$ whose interior contains $a_{1}, \ldots, a_{m}$. 
Remark. As an aside, a closer examination of the argument of [33, pp. 241$242]$ shows that one can take $m=\operatorname{dim}([\mathfrak{g}, \mathfrak{g}])$, and that $a_{1}, \ldots, a_{m}$ could be chosen arbitrarily chose to $e$; thus the conclusion of Lemma 3.1 actually holds for any neighborhood $U^{\prime}$ of $e$ in $G$.

Next, we need the following structural result for $G_{1}$.

Lemma 3.2 There exists a compact, connected, abelian subgroup $C$ of $G$, with Lie algebra $\mathfrak{c}$, such that $\mathfrak{g}_{1}=\mathfrak{c}+[\mathfrak{g}, \mathfrak{g}]$ and $G_{1}=C[G, G]$. (Here, + denotes a sum of vector subspaces which is not necessarily direct.)

If $U^{\prime}, U^{\prime \prime}, m$ are as in Lemma 3.1, then $U_{1}:=C\left(U^{\prime \prime}\right)^{m}$ is a neighborhood of the identity for the group $G_{1}$.

Proof. The existence of $C$ with the properties $\mathfrak{g}_{1}=\mathfrak{c}+[\mathfrak{g}, \mathfrak{g}], G_{1}=C[G, G]$, is proved in the Appendix of [15] (see also [37, Appendix] for similar results when $G$ is amenable). That $C\left(U^{\prime \prime}\right)^{m}$ is a neighborhood of the identity for $G_{1}$ then follows from Lemma 3.1.

In the rest of this section, we shall fix $U^{\prime}, U^{\prime \prime}, m, C$ and $U_{1}=C\left(U^{\prime \prime}\right)^{m}$ with properties as in Lemmas 3.1 and 3.2.

Lemma 3.3 There exists $c>0$ such that $\left|\left(\partial_{g} f_{1}, f_{2}\right)\right| \leq c \Gamma_{2}\left(f_{1}\right) \Gamma_{2}\left(f_{2}\right)$ for all $g \in U^{\prime \prime}$ and $f_{1}, f_{2} \in L^{2}$.

Proof. Let $g=\left[g_{1}, g_{2}\right] \in U^{\prime \prime}$ where $g_{1}, g_{2} \in U^{\prime}$. The identity

$$
\partial_{g}=-L\left(g_{1} g_{2}\right)\left(\partial_{g_{2}^{-1}} \partial_{g_{1}^{-1}}-\partial_{g_{1}^{-1}} \partial_{g_{2}^{-1}}\right)
$$

yields

$$
\left(\partial_{g} f_{1}, f_{2}\right)=-\left(\partial_{g_{1}^{-1}} f_{1}, \partial_{g_{2}} L\left(g_{2}^{-1} g_{1}^{-1}\right) f_{2}\right)+\left(\partial_{g_{2}^{-1}} f_{1}, \partial_{g_{1}} L\left(g_{2}^{-1} g_{1}^{-1}\right) f_{2}\right) .
$$

We use the Cauchy-Schwarz inequality, (2.5), and (2.6) to obtain

$$
\begin{aligned}
\left|\left(\partial_{g} f_{1}, f_{2}\right)\right| & \leq c \Gamma_{2}\left(f_{1}\right) \Gamma_{2}\left(L\left(g_{2}^{-1} g_{1}^{-1}\right) f_{2}\right) \\
& \leq c^{\prime} \Gamma_{2}\left(f_{1}\right) \Gamma_{2}\left(f_{2}\right)
\end{aligned}
$$

for all $g \in U^{\prime \prime}$ and $f_{1}, f_{2} \in L^{2}$.

Lemma 3.4 There exists $c>0$ such that $\left|\left(\partial_{g} f_{1}, f_{2}\right)\right| \leq c \Gamma_{2}\left(f_{1}\right) \Gamma_{2}\left(f_{2}\right)$ for all $g \in U_{1}$ and $f_{1}, f_{2} \in L^{2}$. 
Proof. Let $d s$ be Haar measure on the compact group $C$, normalized so that $d s(C)=1$. For any $s_{0} \in C$, we have the operator identity

$$
\partial_{s_{0}}=-\int_{C} d s \partial_{s} \partial_{s_{0}}
$$

Using (2.5) we deduce that

$$
\left|\left(\partial_{s_{0}} f_{1}, f_{2}\right)\right| \leq \int_{C} d s\left|\left(\partial_{s_{0}} f_{1}, \partial_{s^{-1}} f_{2}\right)\right| \leq c \Gamma_{2}\left(f_{1}\right) \Gamma_{2}\left(f_{2}\right)
$$

for all $s_{0} \in C$. Next, for $g \in U_{1}=C\left(U^{\prime \prime}\right)^{m}$ we write $g=s_{0} g_{1} g_{2} \cdots g_{m}$ with $s_{0} \in C$ and $g_{j} \in U^{\prime \prime}$. Applying (2.3) shows that

$$
\left(\partial_{g} f_{1}, f_{2}\right)=\left(\partial_{s_{0}} f_{1}, f_{2}\right)+\sum_{j=1}^{m}\left(\partial_{g_{j}} f_{1}, L\left(\left(s_{0} g_{1} \cdots g_{j-1}\right)^{-1}\right) f_{2}\right) .
$$

The lemma follows by applying the above estimate for $\partial_{s_{0}}$, the estimate of Lemma 3.3, and (2.6).

In order to extend the estimate of Lemma 3.4, observe that $G_{1}=\bigcup_{n=1}^{\infty} U_{1}^{n}$ since $U_{1}$ is a neighborhood of $e$ for the connected group $G_{1}$. We may therefore define a distance $\rho_{1}: G_{1} \rightarrow \mathbb{N}$ by

$$
\rho_{1}(g)=\inf \left\{n \in \mathbb{N}: g \in U_{1}^{n}\right\}, \quad g \in G_{1} .
$$

From $G_{1} \subseteq G$ it follows easily that $\rho(g) \leq c \rho_{1}(g)$ for some constant $c>0$ and all $g \in G_{1}$. On the other hand, a theorem of Varopoulos [36] asserts that any closed connected subgroup of a connected Lie group has at most exponential distance distortion. Applied to $G_{1} \subseteq G$, this means that there is $c^{\prime}>0$ such that

$$
\rho_{1}(g) \leq c^{\prime} e^{c^{\prime} \rho(g)}, \quad g \in G_{1}
$$

We can now prove a version of (3.1).

Lemma 3.5 There exist $c, c^{\prime}>0$ such that

$$
\left|\left(\partial_{g} f_{1}, f_{2}\right)\right| \leq c e^{c^{\prime} \rho(g)} \Gamma_{2}\left(f_{1}\right) \Gamma_{2}\left(f_{2}\right)
$$

for all $g \in G_{1}$ and $f_{1}, f_{2} \in L^{2}$. 
Proof. Let $g \in G_{1}$ and set $n=\rho_{1}(g)$. Then $g=g_{1} g_{2} \cdots g_{n}$ for some $g_{j} \in U_{1}$. From (2.3) we have

$$
\left(\partial_{g} f_{1}, f_{2}\right)=\left(\partial_{g_{1}} f_{1}, f_{2}\right)+\sum_{j=2}^{n}\left(\partial_{g_{j}} f_{1}, L\left(\left(g_{1} \cdots g_{j-1}\right)^{-1}\right) f_{2}\right) .
$$

Hence by Lemma 3.4 and (2.6),

$$
\begin{aligned}
\left|\left(\partial_{g} f_{1}, f_{2}\right)\right| & \leq c \Gamma_{2}\left(f_{1}\right) \Gamma_{2}\left(f_{2}\right)+c \sum_{j=2}^{n} \Gamma_{2}\left(f_{1}\right) \Gamma_{2}\left(L\left(\left(g_{1} \cdots g_{j-1}\right)^{-1}\right) f_{2}\right) \\
& \leq c \Gamma_{2}\left(f_{1}\right) \Gamma_{2}\left(f_{2}\right)+c^{\prime} \sum_{j=2}^{n} \rho\left(g_{1} \ldots g_{j-1}\right) \Gamma_{2}\left(f_{1}\right) \Gamma_{2}\left(f_{2}\right) .
\end{aligned}
$$

Since

$$
\rho\left(g_{1} \cdots g_{j-1}\right) \leq \sum_{i=1}^{j-1} \rho\left(g_{i}\right) \leq c^{\prime \prime}(j-1) \leq c^{\prime \prime} n
$$

where $c^{\prime \prime}=\sup \left\{\rho(g): g \in U_{1}\right\}$, this yields an estimate

$$
\left|\left(\partial_{g} f_{1}, f_{2}\right)\right| \leq c n^{2} \Gamma_{2}\left(f_{1}\right) \Gamma_{2}\left(f_{2}\right) .
$$

Recalling that $n=\rho_{1}(g)$, the lemma follows using (3.3).

Proof of Theorem 1.3. Recall that $\pi_{1}: G \rightarrow G / G_{1} \cong \mathbb{R}^{q}$; we identify $G / G_{1}$ with $\mathbb{R}^{q}$ so that $\pi_{1}(g)=\left(\pi_{1}^{(1)}(g), \ldots, \pi_{1}^{(q)}(g)\right) \in \mathbb{R}^{q}, g \in G$.

Let $\partial /\left(\partial y_{1}\right), \ldots, \partial /\left(\partial y_{q}\right)$ be the standard coordinate vector fields on $\mathbb{R}^{q}$, and fix $X_{1}, \ldots, X_{q} \in \mathfrak{g}$ such that $\left(\pi_{1}\right)_{*} X_{j}=\partial /\left(\partial y_{j}\right)$ for all $j$. Let $\exp : \mathfrak{g} \rightarrow G$ be the exponential map, and define the one-parameter subgroups $x_{j}: \mathbb{R} \rightarrow G$ by $x_{j}(t):=\exp \left(t X_{j}\right)$ for $t \in \mathbb{R}, j \in\{1, \ldots, q\}$.

Given any $g \in G$, put $t_{j}:=\pi_{1}^{(j)}(g) \in \mathbb{R}, j \in\{1, \ldots, q\}$, and write

$$
g=x_{1}\left(t_{1}\right) \cdots x_{q}\left(t_{q}\right) g^{\prime}
$$

for some $g^{\prime} \in G$. Because $\pi_{1}(g)=\left(t_{1}, \ldots, t_{q}\right)=\pi_{1}\left(x_{1}\left(t_{1}\right) \cdots x_{q}\left(t_{q}\right)\right)$ and $G_{1}$ is the kernel of the homomorphism $\pi_{1}$, then $g^{\prime} \in G_{1}$. In what follows, $c, c^{\prime}$ will denote constants independent of $g \in G$. Since $\pi_{1}^{(j)}: G \rightarrow \mathbb{R}$ and $x_{j}: \mathbb{R} \rightarrow G$ are homomorphisms, it is straightforward to establish inequalities of form

$$
\begin{aligned}
\left|t_{j}\right| & =\left|\pi_{1}^{(j)}(g)\right| \leq c \rho(g), \\
\sup _{|s| \leq\left|t_{j}\right|} \rho\left(x_{j}(s)\right) & \leq c \rho(g), \quad \sup _{|u| \leq 1} \rho\left(x_{j}(u)\right) \leq c, \\
\rho\left(g^{\prime}\right) & \leq c \rho(g),
\end{aligned}
$$

for all $g \in G$. 
Applying identity (2.4) to (3.4) shows that

$$
\partial_{g}=\partial_{x_{1}\left(t_{1}\right)}+\cdots+\partial_{x_{q}\left(t_{q}\right)}+\partial_{g^{\prime}}+W_{g}
$$

where the operator $W_{g}$ is a finite sum of terms each of the form $\partial_{h_{1}} \cdots \partial_{h_{k}}$ with $k \geq 2$ and $h_{1}, \ldots, h_{k} \in\left\{x_{1}\left(t_{1}\right), \ldots, x_{q}\left(t_{q}\right), g^{\prime}\right\}$. By writing

$$
\begin{aligned}
\left|\left(\partial_{h_{1}} \ldots \partial_{h_{k}} f_{1}, f_{2}\right)\right| & =\left|\left(\partial_{h_{2}} \ldots \partial_{h_{k}} f_{1}, \partial_{h_{1}^{-1}} f_{2}\right)\right| \\
& \leq\left\|\partial_{h_{2}} \ldots \partial_{h_{k}} f_{1}\right\|_{2}\left\|\partial_{h_{1}^{-1}} f_{2}\right\|_{2} \leq 2^{k-2}\left\|\partial_{h_{k}} f_{1}\right\|_{2}\left\|\partial_{h_{1}^{-1}} f_{2}\right\|_{2},
\end{aligned}
$$

one sees from (2.5) and (3.5) that

$$
\left|\left(W_{g} f_{1}, f_{2}\right)\right| \leq c \rho(g)^{2} \Gamma_{2}\left(f_{1}\right) \Gamma_{2}\left(f_{2}\right)
$$

for all $f_{1}, f_{2} \in L^{2}$. Also, since $g^{\prime} \in G_{1}$, Lemma 3.5 and (3.5) provide an estimate

$$
\left|\left(\partial_{g^{\prime}} f_{1}, f_{2}\right)\right| \leq c e^{c \rho\left(g^{\prime}\right)} \Gamma_{2}\left(f_{1}\right) \Gamma_{2}\left(f_{2}\right) \leq c e^{c^{\prime} \rho(g)} \Gamma_{2}\left(f_{1}\right) \Gamma_{2}\left(f_{2}\right)
$$

for $f_{1}, f_{2} \in L^{2}$. Next, the following lemma allows us to compare $\partial_{x_{j}\left(t_{j}\right)}$ and $t_{j} \partial_{x_{j}(1)}$.

Lemma 3.6 Let $\left(R_{t}\right)_{t \in \mathbb{R}} \subseteq \mathcal{L}(X)$ be a strongly continuous one-parameter group of bounded linear operators in a Banach space $X$. Set $D_{t}:=R_{t}-I$ for $t \in \mathbb{R}$. Then

$$
D_{t}-t D_{1}=-\int_{0}^{1} d s D_{s} D_{t}+\int_{0}^{t} d s D_{s} D_{1}
$$

where the integrals converge with respect to the strong operator topology.

Proof of Lemma 3.6. For $t \in \mathbb{R}$ set

$$
\begin{aligned}
P_{t} & :=\int_{0}^{t} d s\left(R_{s+1}-R_{s}\right) \\
& =\int_{1}^{t+1} d s R_{s}-\int_{0}^{t} d s R_{s}=\int_{0}^{1} d s\left(R_{s+t}-R_{s}\right),
\end{aligned}
$$

and observe, using the group property $R_{u} R_{v}=R_{u+v}$, that

$$
\begin{aligned}
& D_{t}-P_{t}=\int_{0}^{1} d s\left(D_{t}-\left(R_{s+t}-R_{s}\right)\right)=-\int_{0}^{1} d s D_{s} D_{t}, \\
& P_{t}-t D_{1}=\int_{0}^{t} d s\left(\left(R_{s+1}-R_{s}\right)-D_{1}\right)=\int_{0}^{t} d s D_{s} D_{1} .
\end{aligned}
$$

The lemma follows. 
Setting $R_{t}=L\left(x_{j}(t)\right)$ and $D_{t}=\partial_{x_{j}(t)}$ in Lemma 3.6 gives

$$
\partial_{x_{j}\left(t_{j}\right)}-t_{j} \partial_{x_{j}(1)}=-\int_{0}^{1} d s \partial_{x_{j}(s)} \partial_{x_{j}\left(t_{j}\right)}+\int_{0}^{t_{j}} d s \partial_{x_{j}(s)} \partial_{x_{j}(1)} .
$$

From this identity and (2.5), (3.5), one easily sees that

$$
\left|\left(\left(\partial_{x_{j}\left(t_{j}\right)}-t_{j} \partial_{x_{j}(1)}\right) f_{1}, f_{2}\right)\right| \leq c \rho(g)^{2} \Gamma_{2}\left(f_{1}\right) \Gamma_{2}\left(f_{2}\right)
$$

for $f_{1}, f_{2} \in L^{2}$.

By combining (3.7), (3.8), (3.9) with (3.6) and recalling that $t_{j}=\pi_{1}^{(j)}(g)$, we conclude that

$$
\partial_{g}=\sum_{j=1}^{q} \pi_{1}^{(j)}(g) \partial_{x_{j}(1)}+V_{g}
$$

where the linear operator $V_{g} \in \mathcal{L}\left(L^{2}\right)$ satisfies

$$
\left|\left(V_{g} f_{1}, f_{2}\right)\right| \leq c e^{c \rho(g)} \Gamma_{2}\left(f_{1}\right) \Gamma_{2}\left(f_{2}\right)
$$

for all $g \in G, f_{1}, f_{2} \in L^{2}$. But since $K$ is centered, it follows from (1.1) and (1.4) that

$$
(I-T) f=-\int_{G} d g K(g) \partial_{g} f=-\int_{G} d g K(g) V_{g} f
$$

for all $f \in L^{2}$. Therefore, by (3.11) and Assumption (ii),

$$
\begin{aligned}
|((I-T) f, f)| & \leq \int_{G} d g K(g)\left|\left(V_{g} f, f\right)\right| \\
& \leq \int_{G} d g K(g) c e^{c \rho(g)} \Gamma_{2}(f)^{2} \leq c^{\prime} \Gamma_{2}(f)^{2}
\end{aligned}
$$

for all $f \in L^{2}$. This ends the proofs of Theorems 1.3 and 1.2.

Remarks. (a) Assumption (ii) of Theorem 1.2 can be relaxed in case $G$ has polynomial volume growth. Indeed, in this case, by a result of [36] (see also [7]) any closed subgroup of $G$ has polynomial distance distortion, which means that there exists $N \geq 1$ with

$$
\rho_{1}(g) \leq c \rho(g)^{N}
$$

for all $g \in G_{1}$. Then, one can prove that $T$ is analytic for any centered density $K$ satisfying Assumption (i) and the condition

$$
\int_{G} d g K(g) \rho(g)^{2 N}<\infty
$$


This result follows by an easy adaption of the above proofs; one uses (3.12) to obtain Lemma 3.5 in the form

$$
\left|\left(\partial_{g} f_{1}, f_{2}\right)\right| \leq c \rho(g)^{2 N} \Gamma_{2}\left(f_{1}\right) \Gamma_{2}\left(f_{2}\right)
$$

for all $f_{i} \in L^{2}$ and $g \in G_{1}$.

(b) Let us describe an $L^{p}$ version of the $L^{2}$ estimate of Theorem 1.3.

Fix $p \in[1, \infty]$ with $p^{-1}+\left(p^{\prime}\right)^{-1}=1$ and consider the pairing $\left(f_{1}, f_{2}\right):=$ $\int_{G} d g f_{1} \overline{f_{2}}$ for $f_{1} \in L^{p}, f_{2} \in L^{p^{\prime}}$. By modifying the arguments leading to $(3.11)$, one obtains

$$
\left|\left(V_{g} f_{1}, f_{2}\right)\right| \leq c e^{c \rho(g)} \Gamma_{p}\left(f_{1}\right) \Gamma_{p^{\prime}}\left(f_{2}\right)
$$

for all $g \in G, f_{1} \in L^{p}, f_{2} \in L^{p^{\prime}}$, where $V_{g}$ is as in (3.10). To see this, one uses Hölder's inequality in place of the Cauchy-Schwarz inequality in the proofs of Lemmas 3.3, 3.4, 3.5 and Theorem 1.3.

When $K$ is centered and satisfies Assumption (ii), estimate (3.13) yields that

$$
\left|\left((I-T) f_{1}, f_{2}\right)\right|=\left|\int_{G} d g K(g)\left(V(g) f_{1}, f_{2}\right)\right| \leq c \Gamma_{p}\left(f_{1}\right) \Gamma_{p^{\prime}}\left(f_{2}\right)
$$

for all $f_{1} \in L^{p}$ and $f_{2} \in L^{p^{\prime}}$. In particular, with $p=\infty$ we get that

$$
|((I-T) \psi, f)| \leq c \Gamma_{1}(f)
$$

for all $\psi \in \mathcal{E} \cap L^{\infty}$ and $f \in L^{1}$. Because the right side of (3.15) is independent of $\|\psi\|_{\infty}$, an easy approximation argument then gives (3.15) for all $\psi \in \mathcal{E}$.

The estimate (3.15) for centered $K$ will be crucial for the proof of Theorem 1.5.

\section{Proof of Theorem 1.5}

For the proof of Theorem 1.5, in this section $K$ will denote a sub-Gaussian density on the connected Lie group $G$. Let us fix $\psi \in \mathcal{E}$ and set $T_{\lambda}:=$ $e^{\lambda \psi} T e^{-\lambda \psi}$ for $\lambda \in \mathbb{R}$. The constants in the estimates obtained below do not, however, depend on the particular choice of $\psi \in \mathcal{E}$.

Note that, by (2.5), we have $\left\|\partial_{g} \psi\right\|_{\infty} \leq \rho(g)$ for all $g \in G$.

We begin with a simple estimate of $T_{\lambda}-T$ which is valid whether or not $K$ is centered.

Lemma 4.1 There exist constants $c, \omega>0$ such that $\left\|T_{\lambda}-T\right\|_{p \rightarrow p} \leq c|\lambda| e^{\omega \lambda^{2}}$ for all $\lambda \in \mathbb{R}$ and $p \in[1, \infty]$. 
Proof. Note the identity

$$
e^{\lambda \psi} L(g)\left(e^{-\lambda \psi} f\right)=e^{-\lambda \partial_{g} \psi} L(g) f
$$

for a function $f: G \rightarrow \mathbb{C}$ and $g \in G$. Since $T=\int_{G} d g K(g) L(g)$, then

$$
\left(T_{\lambda}-T\right) f=\int_{G} d g K(g)\left[e^{-\lambda \partial_{g} \psi}-1\right] L(g) f .
$$

The inequalities $\left|e^{s}-1\right| \leq|s| e^{|s|}, s \in \mathbb{R}$, and $\left\|\partial_{g} \psi\right\|_{\infty} \leq \rho(g)$ imply that

$$
\left\|e^{-\lambda \partial_{g} \psi}-1\right\|_{\infty} \leq|\lambda| \rho(g) e^{|\lambda| \rho(g)}
$$

for all $g \in G, \lambda \in \mathbb{R}$. Note the elementary estimate $|\lambda| \rho(g) \leq \varepsilon \rho(g)^{2}+\varepsilon^{-1} \lambda^{2}$ for all $\varepsilon>0$. Fixing $\varepsilon<b$, where $b$ is as in (1.3), we get

$$
\begin{aligned}
\left\|\left(T_{\lambda}-T\right) f\right\|_{p} & \leq|\lambda| \int_{G} d g K(g) \rho(g) e^{|\lambda| \rho(g)}\|f\|_{p} \\
& \leq c|\lambda| e^{\varepsilon^{-1} \lambda^{2}} \int_{G} d g e^{-(b-\varepsilon) \rho(g)^{2}} \rho(g)\|f\|_{p} \leq c^{\prime}|\lambda| e^{\varepsilon^{-1} \lambda^{2}}\|f\|_{p} .
\end{aligned}
$$

The lemma follows.

Since $\|T\|_{2 \rightarrow 2} \leq 1$, Lemma 4.1 implies that $\left\|T_{\lambda}\right\|_{2 \rightarrow 2}=1+O(|\lambda|)$ for small $|\lambda|$. To prove Theorem 1.5, our main task will be to obtain the improved estimate

$$
\left\|T_{\lambda}\right\|_{2 \rightarrow 2} \leq 1+c \lambda^{2}
$$

for all $|\lambda| \leq 1$, when $K$ is centered. Indeed, given (4.2) and Lemma 4.1 it follows for some $\omega^{\prime}>0$ that $\left\|T_{\lambda}\right\|_{2 \rightarrow 2} \leq e^{\omega^{\prime} \lambda^{2}}$ for all $\lambda \in \mathbb{R}$. This implies $\left\|T_{\lambda}^{n}\right\|_{2 \rightarrow 2} \leq e^{\omega^{\prime} \lambda^{2} n}$ for all $n \in \mathbb{N}$, which is the estimate of Theorem 1.5.

Define quadratic forms $Q$ and $Q_{\lambda}, \lambda \in \mathbb{R}$, by

$$
Q(f)=\|f\|_{2}^{2}-\|T f\|_{2}^{2}, \quad Q_{\lambda}(f)=\|f\|_{2}^{2}-\left\|T_{\lambda} f\right\|_{2}^{2},
$$

for all $f \in L^{2}$, so that $Q_{0}=Q$. To obtain (4.2) we require the perturbation estimate of the next proposition. Note that (3.15) is essential for the proof of the proposition.

Proposition 4.2 (I) There exists $c>1$ such that

$$
c^{-1} \Gamma_{2}(f)^{2} \leq Q(f) \leq c \Gamma_{2}(f)^{2}
$$

for all $f \in L^{2}$.

(II) If $K$ is centered, then there exists $c^{\prime}>0$ such that

$$
\left|Q(f)-Q_{\lambda}(f)\right| \leq \varepsilon Q(f)+c^{\prime}\left(1+\varepsilon^{-1}\right) \lambda^{2}\|f\|_{2}^{2}
$$

for all $\varepsilon>0, f \in L^{2}$ and $|\lambda| \leq 1$. 
Proof. First observe that $Q(f)=\left(f,\left(I-T^{*} T\right) f\right)$ and $T^{*} T f=\widetilde{K} * f$, $f \in L^{2}$, where $\widetilde{K}$ is the density on $G$ defined by $\widetilde{K}:=K^{*} * K$ with $K^{*}(g):=$ $\Delta\left(g^{-1}\right) K\left(g^{-1}\right)$. Then part (I) follows easily by applying Lemma 2.5 to $T^{*} T$ in place of $T$.

To prove part (II), it suffices to prove an estimate of form

$$
\left|Q(f)-Q_{\lambda}(f)\right| \leq c|\lambda| \Gamma_{2}(f)\|f\|_{2}+c \lambda^{2}\|f\|_{2}^{2}
$$

for all $f \in L^{2},|\lambda| \leq 1$. For then part (II) follows from part (I) and the elementary estimate $|\lambda| Q(f)^{1 / 2}\|f\|_{2} \leq \varepsilon Q(f)+\varepsilon^{-1} \lambda^{2}\|f\|_{2}^{2}$ for all $\varepsilon>0$.

We begin the proof of (4.3) by writing

$$
\begin{aligned}
Q(f) & -Q_{\lambda}(f)=\left\|T_{\lambda} f\right\|_{2}^{2}-\|T f\|_{2}^{2} \\
& =\left(T f,\left(T_{\lambda}-T\right) f\right)+\left(\left(T_{\lambda}-T\right) f, T f\right)+\left(\left(T_{\lambda}-T\right) f,\left(T_{\lambda}-T\right) f\right) \\
& =2 \operatorname{Re}\left(\left(T_{\lambda}-T\right) f, T f\right)+\left\|\left(T_{\lambda}-T\right) f\right\|_{2}^{2} \\
& =2 \operatorname{Re}\left(\left(T_{\lambda}-T\right) f, f\right)+\left\|\left(T_{\lambda}-T\right) f\right\|_{2}^{2}-2 \operatorname{Re}\left(\left(T_{\lambda}-T\right) f,(I-T) f\right) .
\end{aligned}
$$

By Lemma 4.1, the term $\left\|\left(T_{\lambda}-T\right) f\right\|_{2}^{2}$ on the right side is estimated by $c \lambda^{2}\|f\|_{2}^{2}$ for $|\lambda| \leq 1$. Also, from (1.1) we have $\|(I-T) f\|_{2} \leq c \Gamma_{2}(f)$ so that

$$
\left|\left(\left(T_{\lambda}-T\right) f,(I-T) f\right)\right| \leq c|\lambda|\|f\|_{2} \Gamma_{2}(f)
$$

for all $f \in L^{2}$. To handle the remaining term $\left(\left(T_{\lambda}-T\right) f, f\right)$, by (4.1) we may write

$$
\begin{aligned}
\left(\left(T_{\lambda}-T\right) f, f\right)= & \int_{G} d g K(g)\left(\left[e^{-\lambda \partial_{g} \psi}-1\right] L(g) f, f\right) \\
= & \int_{G} d g K(g)\left(\left[e^{-\lambda \partial_{g} \psi}-1+\lambda \partial_{g} \psi\right] L(g) f, f\right) \\
& -\lambda \int_{G} d g K(g)\left(\left(\partial_{g} \psi\right) \partial_{g} f, f\right) \\
& -\lambda \int_{G} d g K(g)\left(\left(\partial_{g} \psi\right) f, f\right) \\
= & I_{1}+I_{2}+I_{3},
\end{aligned}
$$

using that $L(g) f=\partial_{g} f+f$. We will bound each of the terms $I_{1}, I_{2}, I_{3}$. First, since $\left|e^{s}-1-s\right| \leq s^{2} e^{|s|}$ for $s \in \mathbb{R}$ and $\left\|\partial_{g} \psi\right\|_{\infty} \leq \rho(g),\|L(g) f\|_{2}=\|f\|_{2}$, one obtains

$$
\left|\left(\left[e^{-\lambda \partial_{g} \psi}-1+\lambda \partial_{g} \psi\right] L(g) f, f\right)\right| \leq \lambda^{2} \rho(g)^{2} e^{|\lambda| \rho(g)}\|f\|_{2}^{2}
$$

for all $g \in G$. It easily follows that $\left|I_{1}\right| \leq c \lambda^{2}\|f\|_{2}^{2}$ for all $|\lambda| \leq 1$. 
Next, observing that

$$
\left|\left(\left(\partial_{g} \psi\right) \partial_{g} f, f\right)\right| \leq\left\|\partial_{g} \psi\right\|_{\infty}\left\|\partial_{g} f\right\|_{2}\|f\|_{2} \leq \rho(g)^{2} \Gamma_{2}(f)\|f\|_{2}
$$

we deduce that $\left|I_{2}\right| \leq c|\lambda| \Gamma_{2}(f)\|f\|_{2}$ for all $|\lambda| \leq 1$.

Finally, from (1.1) we can write

$$
I_{3}=\lambda(((I-T) \psi) f, f)=\lambda((I-T) \psi, f \bar{f}) .
$$

Since $K$ is centered (this is the only place in the argument where centeredness is used), (3.15) gives

$$
|((I-T) \psi, f \bar{f})| \leq c \Gamma_{1}(f \bar{f}) \leq 2 c \Gamma_{2}(f)\|f\|_{2}
$$

for all $f \in L^{2}$, where the second inequality follows easily from the identity $\partial_{u}\left(f_{1} f_{2}\right)=\left(\partial_{u} f_{1}\right) f_{2}+\left(L(u) f_{1}\right)\left(\partial_{u} f_{2}\right)$ for $u \in U, f_{1}, f_{2} \in L^{2}$. Thus $\left|I_{3}\right| \leq$ $c^{\prime}|\lambda| \Gamma_{2}(f)\|f\|_{2}$ for all $|\lambda| \leq 1$. Collecting the above estimates yields (4.3), and Proposition 4.2 is proved.

Remark. The proof of Proposition 4.2 also yields the following perturbation estimate of interest: for centered $K$, one has

$$
\left|\left(\left(T_{\lambda}-T\right) f, f\right)\right| \leq \varepsilon \Gamma_{2}(f)^{2}+c\left(1+\varepsilon^{-1}\right) \lambda^{2}\|f\|_{2}^{2}
$$

for all $\varepsilon>0,|\lambda| \leq 1$ and $f \in L^{2}$. Actually, our argument above shows that (4.4) is essentially equivalent with the estimate of Proposition 4.2, Part (II).

To continue with the proof of $(4.2)$, choose $\varepsilon=1 / 2$ in the estimate of Proposition 4.2, part (II). We find, for some $c>0$, that

$$
\begin{aligned}
\|f\|_{2}^{2}-\left\|T_{\lambda} f\right\|_{2}^{2} & =Q_{\lambda}(f) \geq Q(f)-\left|Q(f)-Q_{\lambda}(f)\right| \\
& \geq 2^{-1} Q(f)-c \lambda^{2}\|f\|_{2}^{2} \geq-c \lambda^{2}\|f\|_{2}^{2}
\end{aligned}
$$

for all $f \in L^{2}$ and $|\lambda| \leq 1$. Thus $\left\|T_{\lambda} f\right\|_{2}^{2} \leq\left(1+c \lambda^{2}\right)\|f\|_{2}^{2}$ and $\left\|T_{\lambda}\right\|_{2 \rightarrow 2} \leq$ $\left(1+c \lambda^{2}\right)^{1 / 2} \leq 1+c \lambda^{2}$ for $|\lambda| \leq 1$. This proves (4.2) and completes the proof of Theorem 1.5.

\section{Proof of Theorem 1.6}

Theorem 1.6 essentially follows from Theorem 1.5 and the equivalence between two different forms of $L^{p}$ off-diagonal estimates.

On any locally compact, compactly generated group $G$, we can give the following precise statement of this equivalence. (If $E$ or $F$ is the empty set, adopt the convention that $d(E, F)=+\infty$ and $e^{-b d(E, F)^{2}}=0$ for $b>0$.) 
Proposition 5.1 Fix $p \in[1, \infty]$, let $S \in \mathcal{L}\left(L^{p}\right)$ and let $n \in \mathbb{N}$.

(I) If there exist constants $a, \omega>0$ such that

$$
\left\|e^{\lambda \psi} S e^{-\lambda \psi}\right\|_{p \rightarrow p} \leq a e^{\omega \lambda^{2} n}
$$

for all $\lambda \in \mathbb{R}$ and $\psi \in \mathcal{E}$, then there exist $c, b>0$ depending on $\omega$ but not on $n$ or $a$, such that

$$
\left\|\chi_{E} S \chi_{F}\right\|_{p \rightarrow p} \leq c a e^{-b d(E, F)^{2} / n}
$$

for all Borel measurable subsets $E, F \subseteq G$.

(II) Conversely, if there exist $a, b>0$ such that

$$
\left\|\chi_{E} S \chi_{F}\right\|_{p \rightarrow p} \leq a e^{-b d(E, F)^{2} / n}
$$

for all Borel measurable $E, F \subseteq G$, then there exist $c, \omega>0$ depending on $b$ but not on $n$ or a, such that

$$
\left\|e^{\lambda \psi} S e^{-\lambda \psi}\right\|_{p \rightarrow p} \leq c a e^{\omega \lambda^{2} n}
$$

for all $\lambda \in \mathbb{R}$ and $\psi \in \mathcal{E}$.

The case $p=2$ of Theorem 1.6 is an immediate consequence of Theorem 1.5 and Proposition 5.1. The general case $p \in(1, \infty)$ of Theorem 1.6 then follows by interpolation between the case $p=2$ and the obvious estimates $\left\|\chi_{E} T^{n} \chi_{F}\right\|_{1 \rightarrow 1} \leq\left\|T^{n}\right\|_{1 \rightarrow 1} \leq 1,\left\|\chi_{E} T^{n} \chi_{F}\right\|_{\infty \rightarrow \infty} \leq 1$.

Thus it only remains to prove Proposition 5.1, and the rest of this section is occupied with this proof.

The proof of part (I) is a variation of standard ideas (see for example [13].) To prove part (I), let $E, F$ be non-empty with $d(E, F) \geq 2$ (if $d(E, F)=1$ the desired estimate is trivial since $\left.\left\|\chi_{E} S \chi_{F}\right\|_{p \rightarrow p} \leq\|S\|_{p \rightarrow p} \leq a.\right)$ We may assume that $a=1$ (otherwise, replace $S$ by $a^{-1} S$ ). Define $\psi_{F}: G \rightarrow \mathbb{N}$ by $\psi_{F}(g)=d(\{g\}, F)=\inf \left\{\rho\left(g h^{-1}\right): h \in F\right\}$. The bound

$$
\left|\psi_{F}(h)-\psi_{F}(g h)\right| \leq \rho(g), \quad g, h \in G,
$$

implies that $\psi_{F} \in \mathcal{E}$. For $g \in E$ we have $\psi_{F}(g) \geq d(E, F)$, while for $g \in F$, $\psi_{F}(g)=1 \leq 2^{-1} d(E, F)$. For $\lambda \geq 0$, then

$$
\left\|e^{-\lambda \psi_{F}} \chi_{E}\right\|_{\infty} \leq e^{-\lambda d(E, F)}, \quad\left\|e^{\lambda \psi_{F}} \chi_{F}\right\|_{\infty} \leq e^{2^{-1} \lambda d(E, F)} .
$$

Applying the hypothesis of (I) gives

$$
\begin{aligned}
\left\|\chi_{E} S \chi_{F}\right\|_{p \rightarrow p} & \leq\left\|e^{-\lambda \psi_{F}} \chi_{E}\right\|_{\infty}\left\|e^{\lambda \psi_{F}} S e^{-\lambda \psi_{F}}\right\|_{p \rightarrow p}\left\|e^{\lambda \psi_{F}} \chi_{F}\right\|_{\infty} \\
& \leq e^{\omega \lambda^{2} n-2^{-1} \lambda d(E, F)}
\end{aligned}
$$

for all $\lambda \geq 0$. Choosing $\lambda$ to be a small constant multiple of $d(E, F) / n$ proves part $(\mathrm{I})$. 
For the proof of part (II) we need the following lemma, which actually holds on any measure space.

Lemma 5.2 Let $p, p^{\prime} \in[1, \infty]$ with $p^{-1}+\left(p^{\prime}\right)^{-1}=1$. Let $\left\{E_{k}\right\}$ be a finite or countable sequence of measurable subsets of $G$ such that $G=\bigcup_{k} E_{k}$. Then any linear operator $S$ in $L^{p}$ satisfies

$$
\|S\|_{p \rightarrow p} \leq\left(\sup _{l} \sum_{k}\left\|\chi_{E_{k}} S \chi_{E_{l}}\right\|_{p \rightarrow p}\right)^{1 / p}\left(\sup _{k} \sum_{l}\left\|\chi_{E_{k}} S \chi_{E_{l}}\right\|_{p \rightarrow p}\right)^{1 / p^{\prime}} .
$$

Proof. We may assume that the $E_{k}$ are pairwise disjoint (otherwise, we may replace $\left\{E_{k}\right\}$ with a pairwise disjoint sequence $\left\{E_{k}^{\prime}\right\}$ such that $E_{k}^{\prime} \subseteq E_{k}$ and $\bigcup_{k} E_{k}^{\prime}=\bigcup_{k} E_{k}=G$.) Since the cases $p=1$ or $p=\infty$ are straightforward, we will also assume that $p \in(1, \infty)$. Suppose $\left\|f_{1}\right\|_{p}=1$ and $\left\|f_{2}\right\|_{p^{\prime}}=1$. Set $f_{j}^{(k)}:=\chi_{E_{k}} f_{j}$ for $j=1,2$, and observe that

$$
\left(S f_{1}, f_{2}\right)=\sum_{k, l}\left(\chi_{E_{k}} S \chi_{E_{l}} f_{1}^{(l)}, f_{2}^{(k)}\right) .
$$

By applications of Hölder's inequality, we obtain

$$
\begin{aligned}
& \left|\left(S f_{1}, f_{2}\right)\right| \leq \sum_{k, l}\left\|\chi_{E_{k}} S \chi_{E_{l}}\right\|_{p \rightarrow p}\left\|f_{1}^{(l)}\right\|_{p}\left\|f_{2}^{(k)}\right\|_{p^{\prime}} \\
& =\sum_{k, l}\left(\left\|\chi_{E_{k}} S \chi_{E_{l}}\right\|_{p \rightarrow p}^{1 / p}\left\|f_{1}^{(l)}\right\|_{p}\right)\left(\left\|\chi_{E_{k}} S \chi_{E_{l}}\right\|_{p \rightarrow p}^{1 / p^{\prime}}\left\|f_{2}^{(k)}\right\|_{p^{\prime}}\right) \\
& \leq\left(\sum_{k, l}\left\|\chi_{E_{k}} S \chi_{E_{l}}\right\|_{p \rightarrow p}\left\|f_{1}^{(l)}\right\|_{p}^{p}\right)^{1 / p}\left(\sum_{k, l}\left\|\chi_{E_{k}} S \chi_{E_{l}}\right\|_{p \rightarrow p}\left\|f_{2}^{(k)}\right\|_{p^{\prime}}^{p^{\prime}}\right)^{1 / p^{\prime}} \\
& \leq\left(\sup _{l} \sum_{k}\left\|\chi_{E_{k}} S \chi_{E_{l}}\right\|_{p \rightarrow p}\right)^{1 / p}\left(\sup _{k} \sum_{l}\left\|\chi_{E_{k}} S \chi_{E_{l}}\right\|_{p \rightarrow p}\right)^{1 / p^{\prime}}
\end{aligned}
$$

where the last step used that $\sum_{l}\left\|f_{1}^{(l)}\right\|_{p}^{p}=\left\|f_{1}\right\|_{p}^{p}=1$ and $\sum_{k}\left\|f_{2}^{(k)}\right\|_{p^{\prime}}^{p^{\prime}}=$ $\left\|f_{2}\right\|_{p^{\prime}}^{p^{\prime}}=1$. The lemma follows.

To prove part (II), we may assume that $a=1$. Fix $\psi \in \mathcal{E}$, define

$$
E_{k}:=\left\{g \in G: k n^{1 / 2} \leq \psi(g)<(k+1) n^{1 / 2}\right\}, \quad k \in \mathbb{Z},
$$

and observe that $G$ is the disjoint union of the $E_{k}, k \in \mathbb{Z}$. The conclusion of part (II) will follow from Lemma 5.2, if we can prove a bound

$$
\left\|\chi_{E_{k}} e^{\lambda \psi} S e^{-\lambda \psi} \chi_{E_{l}}\right\|_{p \rightarrow p} \leq c e^{-b^{\prime}|k-l|^{2}} e^{\omega \lambda^{2} n}
$$

for all $\lambda \in \mathbb{R}, k, l \in \mathbb{Z}$, where $c, b^{\prime}, \omega$ are positive constants independent of $n$ and $\psi$. 
We will prove (5.1) for $\lambda \geq 0$ (the case $\lambda \leq 0$ being similar). Since

$$
\left\|\chi_{E_{k}} e^{\lambda \psi}\right\|_{\infty} \leq e^{\lambda(k+1) n^{1 / 2}}, \quad\left\|\chi_{E_{l}} e^{-\lambda \psi}\right\|_{\infty} \leq e^{-\lambda l n^{1 / 2}},
$$

we get

$$
\begin{aligned}
\left\|\chi_{E_{k}} e^{\lambda \psi} S e^{-\lambda \psi} \chi_{E_{l}}\right\|_{p \rightarrow p} & \leq e^{\lambda(|k-l|+1) n^{1 / 2}}\left\|\chi_{E_{k}} S \chi_{E_{l}}\right\|_{p \rightarrow p} \\
& \leq e^{\lambda(|k-l|+1) n^{1 / 2}} e^{-b d\left(E_{k}, E_{l}\right)^{2} / n}
\end{aligned}
$$

for all $\lambda \geq 0$ and $k, l \in \mathbb{Z}$. In case $|k-l| \leq 1$, it follows that

$$
\left\|\chi_{E_{k}} e^{\lambda \psi} S e^{-\lambda \psi} \chi_{E_{l}}\right\|_{p \rightarrow p} \leq e^{2 \lambda n^{1 / 2}} \leq e^{1+\lambda^{2} n}
$$

which implies (5.1) in this case.

In the remaining case where $|k-l| \geq 2$, since $\psi \in \mathcal{E}$ we have

$$
\rho\left(g h^{-1}\right) \geq|\psi(g)-\psi(h)| \geq(|k-l|-1) n^{1 / 2}
$$

whenever $g \in E_{k}$ and $h \in E_{l}$. Hence

$$
d\left(E_{k}, E_{l}\right) \geq(|k-l|-1) n^{1 / 2} \geq 2^{-1}|k-l| n^{1 / 2}
$$

and, by (5.2),

$$
\left\|\chi_{E_{k}} e^{\lambda \psi} S e^{-\lambda \psi} \chi_{E_{l}}\right\|_{p \rightarrow p} \leq e^{2 \lambda|k-l| n^{1 / 2}} e^{-4^{-1} b|k-l|^{2}}
$$

for all $|k-l| \geq 2$ and $\lambda \geq 0$. By writing $2 \lambda|k-l| n^{1 / 2} \leq \varepsilon|k-l|^{2}+\varepsilon^{-1} \lambda^{2} n$ for a small constant $\varepsilon>0$, we deduce a bound (5.1) for $|k-l| \geq 2$. The proof of Proposition 5.1 is complete.

\section{Proof of Theorem 1.7}

The proof of Theorem 1.7 is very similar to the proof of the analogous theorem for discrete groups given in [16, Theorem 1.9]. For this reason, we will only outline the arguments.

The implications (I) $\Rightarrow(\mathrm{III})$ and (III) $\Rightarrow(\mathrm{II})$ follow from the proofs of Theorems 1.3 and 1.2. The implications $(\mathrm{I}) \Rightarrow(\mathrm{V}) \Rightarrow(\mathrm{IV})$ follow from the proof of Theorem 1.5. We also have (I) $\Rightarrow(\mathrm{VI})$ by Theorem 1.6 , and (VI) $\Rightarrow(\mathrm{IV})$ by Proposition 5.1.

It remains to show $(\mathrm{II}) \Rightarrow(\mathrm{I})$ and $(\mathrm{IV}) \Rightarrow(\mathrm{I})$. Suppose that $K$ is a noncentered, sub-Gaussian density on the amenable Lie group $G$. Fix a $j \in$ $\{1, \ldots, q\}$ such that $\int_{G} d g K(g) \pi_{1}^{(j)}(g) \neq 0$. Let $P$ be the image of $K$ under the homomorphism $\pi_{1}^{(j)}: G \rightarrow \mathbb{R}$; that is, $P: \mathbb{R} \rightarrow[0, \infty)$ is the density on $\mathbb{R}$ such that

$$
\int_{\mathbb{R}} d x P(x) F(x)=\int_{G} d g K(g) F\left(\pi_{1}^{(j)}(g)\right)
$$

for all $F \in L^{\infty}(\mathbb{R})$. Then $\int_{\mathbb{R}} d x x P(x) \neq 0$, in other words, $P$ is not centered. 
Let $\psi(x):=x, x \in \mathbb{R}$, and set

$$
S f:=P * f, \quad S_{\lambda} f:=e^{\lambda \psi} S\left(e^{-\lambda \psi} f\right)=\left(e^{\lambda \psi} P\right) * f
$$

for all $f \in L^{p}(\mathbb{R})$ and $\lambda \in \mathbb{R}$. Using the Fourier theory of $L^{2}(\mathbb{R})$, it is straightforward to show that $S$ is not analytic in $L^{2}(\mathbb{R})$ and that the estimate $\left\|S_{\lambda}^{n}\right\|_{2 \rightarrow 2} \leq c e^{\omega \lambda^{2} n}, n \in \mathbb{N}, \lambda \in[-1,1]$, cannot hold. (Compare, for example, $[16$, Lemma 6.2] for similar results for non-centered densities on $\mathbb{Z}$.) But the transference theorem for convolution operators on amenable groups (see [11, Theorem 2.4]) implies that

$$
\left\|(I-S) S^{n}\right\|_{2 \rightarrow 2} \leq\left\|(I-T) T^{n}\right\|_{2 \rightarrow 2}, \quad\left\|S_{\lambda}^{n}\right\|_{2 \rightarrow 2} \leq\left\|e^{\lambda \pi_{1}^{(j)}} T^{n} e^{-\lambda \pi_{1}^{(j)}}\right\|_{2 \rightarrow 2}
$$

for all $n$ and $\lambda$. Consequently, Conditions (II) and (IV) fail when $G$ is amenable and $K$ is not centered.

\section{Further proofs}

In this section we describe the proofs of Theorems 1.8 to 1.13 . We will be brief, since some of the arguments occur elsewhere in a similar form.

Theorem 1.8 follows from Theorem 1.5 and (1.3) via known arguments: compare for example the proof of [15, Theorem 1.2], or [38, pp.126-127]. The idea is to observe that, setting $\widetilde{T}_{\lambda}:=\Delta^{1 / 2} e^{\lambda \rho} T e^{-\lambda \rho} \Delta^{-1 / 2}$, the operators $\widetilde{T}_{\lambda}^{n}$ have integral kernels with respect to the right Haar measure $d \widehat{g}=\Delta\left(g^{-1}\right) d g$ given by

$$
\widetilde{K}_{n, \lambda}(g, h):=e^{\lambda \rho(g)} \Delta^{1 / 2}(g) K^{(n)}\left(g h^{-1}\right) \Delta^{-1 / 2}(h) e^{-\lambda \rho(h)}
$$

for $n \in \mathbb{N}, \lambda \in \mathbb{R}$ and $g, h \in G$. Then, setting $h=e$, the required Gaussian estimate of $K^{(n)}$ follows by optimizing over $\lambda$ in the following bounds:

$$
\begin{aligned}
e^{\lambda(\rho(g)-1)} \Delta^{1 / 2}(g) K^{(n)}(g) & \leq\left\|\widetilde{T}_{\lambda}^{n}\right\|_{\widehat{1} \rightarrow \infty} \\
& \leq\left\|\widetilde{T}_{\lambda}\right\|_{\widehat{2} \rightarrow \infty}\left\|\widetilde{T}_{\lambda}^{n-2}\right\|_{\widehat{2} \rightarrow \widehat{2}}\left\|\widetilde{T}_{\lambda}\right\|_{\widehat{1} \rightarrow \widehat{2}} \leq c e^{\omega \lambda^{2} n}
\end{aligned}
$$

for all $g \in G, n \geq 3$ and $\lambda \in \mathbb{R}$. Here, $\|\cdot\|_{\widehat{p} \rightarrow \widehat{q}}$ denotes the norm of an operator from $L^{p}(G ; d \widehat{g})$ to $L^{q}(G ; d \widehat{g})$. To justify (7.1), note that estimates of type $\left\|\widetilde{T}_{\lambda}^{n}\right\|_{\widehat{2} \rightarrow \widehat{2}} \leq e^{\omega \lambda^{2} n}, n \in \mathbb{N}$, are a consequence of Theorem 1.5 since $\rho \in \mathcal{E}$ and $\Delta^{1 / 2}: L^{2}(G ; d g) \rightarrow L^{2}(G ; d \widehat{g})$ is unitary. The bounds of type $\left\|\widetilde{T}_{\lambda}\right\|_{\widehat{2} \rightarrow \infty}+\left\|\widetilde{T}_{\lambda}\right\|_{\widehat{1} \rightarrow \widehat{2}} \leq c e^{\omega \lambda^{2}}, \lambda \in \mathbb{R}$, follow by integration of (1.3). We omit further details.

Theorem 1.9 is proved in the same way as [16, Theorem 1.11]. In this proof, the estimate $\left\|\left(I-T_{\lambda}\right) T_{\lambda}^{n}\right\|_{2 \rightarrow 2} \leq c n^{-1} e^{\omega \lambda^{2} n}$ (where $T_{\lambda}:=e^{\lambda \psi} T e^{-\lambda \psi}$ ) is deduced from the analyticity of $T$, by applying a perturbation theorem for analytic operators of Blunck [5]. For this deduction one needs the perturbation estimate (4.4). 
Alternatively, a simpler proof of the first estimate of Theorem 1.9 follows from the technique of [17]. In fact, given Theorems 1.2 and 1.6 the argument of [17] yields

$$
\left\|\chi_{E}(I-T) T^{n} \chi_{F}\right\|_{2 \rightarrow 2} \leq c n^{-1} e^{-b d(E, F)^{2} / n}
$$

for all $n \in \mathbb{N}$ and all Borel sets $E, F \subseteq G$. By Proposition 5.1, this bound is equivalent to the desired estimate of $\left\|\left(I-T_{\lambda}\right) T_{\lambda}^{n}\right\|_{2 \rightarrow 2}$.

To obtain the remaining estimates of Theorem 1.9, one observes that

$$
\begin{aligned}
\left\|\partial_{h} f\right\|_{2}^{2} & \leq \rho(h)^{2} \Gamma_{2}(f)^{2} \\
& \leq c^{\prime} \rho(h)^{2} \operatorname{Re}((I-T) f, f) \\
& \leq c \rho(h)^{2}\left[\operatorname{Re}\left(\left(I-T_{\lambda}\right) f, f\right)+c \lambda^{2}\|f\|_{2}^{2}\right]
\end{aligned}
$$

for all $h \in G, f \in L^{2}$ and $|\lambda| \leq 1$, where the last two steps follow from Lemma 2.5 together with (4.4). After replacing $f$ with $T_{\lambda}^{n} f$ and applying the first estimate of Theorem 1.9, this enables one to get the desired estimate of $\left\|\partial_{h} T_{\lambda}^{n}\right\|_{2 \rightarrow 2}$. The similar estimate on

$$
\left\|e^{\lambda \psi} \partial_{h} T^{n} e^{-\lambda \psi}\right\|_{2 \rightarrow 2}=\left\|\left(e^{\lambda \psi} \partial_{h} e^{-\lambda \psi}\right) T_{\lambda}^{n}\right\|_{2 \rightarrow 2}
$$

can then be deduced by a straightforward argument, via the operator identity $e^{\lambda \psi} \partial_{h} e^{-\lambda \psi}=\partial_{h}+\left[e^{-\lambda \partial_{h} \psi}-1\right] L(h)$.

As for Theorem 1.10, the required Gaussian bounds for $K^{(n)}(g)$ and $\partial_{h} K^{(n)}(g)$ follow from (1.3), Theorem 1.5 and Theorem 1.9, by choosing $\psi=\rho \in \mathcal{E}$ and applying [14, Theorem 2.3].

Integration of the Gaussian bound on $K^{(n)}$ in Theorem 1.10 implies an estimate of type

$$
\left\|e^{\lambda \rho} K^{(n)}\right\|_{2} \leq c n^{-D / 4} e^{\omega \lambda^{2} n}
$$

for all $n \in \mathbb{N}$ and $\lambda \geq 0$. Then, one may deduce the desired Gaussian bound of Theorem 1.10 for $K^{(n)}-K^{(n+1)}$, by using the first estimate of Theorem 1.9 together with a factorization

$$
\begin{aligned}
e^{\lambda \rho(g)} & \left|K^{(3 m)}(g)-K^{(3 m+1)}(g)\right| \\
& \leq\left\|e^{\lambda \rho}\left(K^{(2 m)}-K^{(2 m+1)}\right)\right\|_{2}\left\|e^{\lambda \rho} K^{(m)}\right\|_{2} \\
& \leq\left\|e^{\lambda \rho}(I-T) T^{m} e^{-\lambda \rho}\right\|_{2 \rightarrow 2}\left\|e^{\lambda \rho} K^{(m)}\right\|_{2}\left\|e^{\lambda \rho} K^{(m)}\right\|_{2}
\end{aligned}
$$

for $g \in G, m \in \mathbb{N}$ and $\lambda \geq 0$. Here, we applied the identities

$$
K^{(3 m)}-K^{(3 m+1)}=\left(K^{(2 m)}-K^{(2 m+1)}\right) * K^{(m)}
$$

and

$$
K^{(2 m)}-K^{(2 m+1)}=(I-T) T^{m}\left(K^{(m)}\right) .
$$

Then optimize over $\lambda$. (For similar arguments, see [5] and the proof of $[16$, Theorem 1.12].) 
To prove Theorem 1.11, let the centered densities $\left(K_{n}\right)_{n=1}^{\infty}$ and the associated Markov operators $T_{n} f:=K_{n} * f$ be as in the hypothesis. Since the $K_{n}$ satisfy the assumptions uniformly in $n$, an inspection of the proofs of Sections 3 and 4 shows that the estimate

$$
\left\|e^{\lambda \psi} T_{n} e^{-\lambda \psi}\right\|_{2 \rightarrow 2} \leq e^{\omega \lambda^{2}}
$$

holds uniformly for all $n \in \mathbb{N}, \lambda \in \mathbb{R}$ and $\psi \in \mathcal{E}$. To see this, one observes that (3.15) and the estimates of Lemma 4.1 and Proposition 4.2 hold with $T_{n}$ replacing $T$, with constants independent of $n$.

Now (1.7) is an immediate consequence of (7.2). The Gaussian bound (1.8) then follows by the same technique used to prove Theorem 1.8.

We next outline the proof of Theorem 1.12. The uniform estimate

$$
\left\|K_{m+n} * \cdots * K_{m+1}\right\|_{\infty} \leq c n^{-D / 2}
$$

for all $m \in \mathbb{N}_{0}, n \in \mathbb{N}$, follows from the proof of [38, Theorem VII.1.2]. Together with the bounds (1.7), this enables one to run the argument of $[22$, Lemma 2.2] to derive the Gaussian bound of Theorem 1.12. We omit further details.

Finally, let us prove Theorem 1.13. With notation as in the theorem, consider the "numerical range"

$$
\Theta_{\alpha}:=\left\{\left(T_{(\alpha)} f, f\right): f \in L^{2},\|f\|_{2}=1\right\} \subseteq \mathbb{C}
$$

of $T_{(\alpha)}$ for each $\alpha \in A$. Because of the uniform assumptions on the $K_{(\alpha)}$, an inspection of the proofs of Sections 2 and 3 shows that estimate (2.2) holds for $T_{(\alpha)}$ with a constant $c>0$ independent of $\alpha$. This means that for some constant $\theta \in(0, \pi / 2)$ one has

$$
\Theta_{\alpha} \subseteq\{\lambda \in \mathbb{C}:|\arg (1-\lambda)| \leq \theta\}
$$

for all $\alpha \in A$. Similarly, the proof of Proposition 2.2 yields for some $\delta \in(0,1]$ that $\Theta_{\alpha} \subseteq \Lambda_{\delta}$ for all $\alpha \in A$, where $\Lambda_{\delta}$ is defined as in (2.1). Hence

$$
\Theta_{\alpha} \subseteq \Gamma_{\delta, \theta}:=\Lambda_{\delta} \cap\{\lambda \in \mathbb{C}:|\arg (1-\lambda)| \leq \theta\},
$$

and from Lemma 2.3 we infer a resolvent estimate

$$
\left\|\left(\lambda I-T_{(\alpha)}\right)^{-1}\right\|_{2 \rightarrow 2} \leq\left(\operatorname{dist}\left(\lambda, \Gamma_{\delta, \theta}\right)\right)^{-1}
$$

uniformly for all $\lambda \in \mathbb{C} \backslash \Gamma_{\delta, \theta}$ and $\alpha \in A$. Using (7.3), one may check that the argument of $\left[29\right.$, p.102] applies uniformly in $\alpha$, to give $\left\|\left(I-T_{(\alpha)}\right) T_{(\alpha)}^{n}\right\|_{2 \rightarrow 2} \leq$ $c n^{-1}$ for all $\alpha \in A$ and $n \in \mathbb{N}$. (The argument of [29] is essentially the proof of the implication (III) $\Rightarrow$ (I) of Theorem 2.1.) This establishes Theorem 1.13 when $p=2$.

Then, thanks to (7.3), one can verify that the proof of the interpolation theorem for analytic operators ([6, Theorem 1.1]) goes through uniformly in $\alpha$, to yield Theorem 1.13 for $p \in(1, \infty)$. We omit further details. 


\section{Sublaplacians}

In this section, we prove Theorems 1.14 and 1.15 .

Proof of Theorem 1.14. Let

$$
H=-\sum_{i=1}^{d^{\prime}} A_{i}^{2}+A_{0}
$$

be a sublaplacian with drift on the connected Lie group $G$, and write $S_{t}:=$ $e^{-t H}$ and $S_{t} f=K_{t} * f$ for $t>0$. It is well known that $(t, g) \mapsto K_{t}(g)$ is a strictly positive, $C^{\infty}$ function on $(0, \infty) \times G$. Moreover, $K_{t}$ is a sub-Gaussian density on $G$ for each fixed $t>0$ (see, for example, [35, Appendix A.4]), and one has the following estimates. Given a compact interval $\left[\delta_{1}, \delta_{2}\right] \subseteq(0, \infty)$, $k, m \in \mathbb{N}_{0}=\{0,1,2, \ldots\}$ and any right invariant vector fields $X_{1}, \ldots, X_{m}$, then there exist $c, b>0$ with

$$
\left|(d / d t)^{k} X_{1} \ldots X_{m} K_{t}(g)\right| \leq c e^{-b \rho(g)^{2}}
$$

for all $t \in\left[\delta_{1}, \delta_{2}\right]$ and $g \in G$. In fact, (8.1) follows from the sub-Gaussian property by applying a local parabolic Harnack inequality of the type given in [38, Theorem III.2.1].

Assuming that $H$ is centered, one easily checks that $A_{0} \pi_{1}^{(j)}=0$ and $H \pi_{1}^{(j)}=0$ for all $j \in\{1, \ldots, q\}$. It is straightforward to deduce that $K_{t} *$ $\pi_{1}^{(j)}=\pi_{1}^{(j)}$, which implies that $K_{t}$ is a centered density for each $t>0$.

These observations imply that the family of densities $\left\{K_{u}: 1 \leq u \leq 2\right\}$ satisfies the hypotheses of Theorem 1.13. Fix $p \in(1, \infty)$. Theorem 1.13 gives an estimate

$$
\left\|\left(I-S_{u}\right)\left(S_{u}\right)^{n}\right\|_{p \rightarrow p}=\left\|\left(I-S_{u}\right) S_{n u}\right\|_{p \rightarrow p} \leq c n^{-1}
$$

for all $n \in \mathbb{N}$ and $u \in[1,2]$. Since $\left\|S_{t}\right\|_{p \rightarrow p} \leq 1$ for all $t$, it is easy to deduce that

$$
\left\|\left(I-S_{s}\right) S_{t}\right\|_{p \rightarrow p} \leq c^{\prime} t^{-1}
$$

for all $t \geq 2^{-1}$ and $s \in[0,1]$. Note that (8.1) implies that $H S_{s}=-(d / d s) S_{s}$ is bounded in $L^{p}$ for every $s>0$. By writing $I-S_{1}=\int_{0}^{1} d s H S_{s}$ and

$$
H S_{t}=\left(I-S_{1}\right) S_{t}+H S_{1 / 2} \int_{0}^{1} d s\left(I-S_{s}\right) S_{t-(1 / 2)}
$$

we obtain

$$
\left\|H S_{t}\right\|_{p \rightarrow p} \leq c t^{-1}+c^{\prime}\left\|H S_{1 / 2}\right\|_{p \rightarrow p} t^{-1} \leq c^{\prime \prime} t^{-1}
$$

for all $t \geq 1$. Theorem 1.14 is proved. 
Remark. The above proof establishes the following abstract result. Let $S_{t}=e^{-t H}, t \geq 0$, be a uniformly bounded semigroup of operators in a Banach space. Suppose (8.2) holds for all $s \in[0,1]$ and $t \geq t_{0}$, for some constant $t_{0}>0$, and that $H S_{u}$ is bounded for some $u>0$. Then $\left\|H S_{t}\right\| \leq$ $c t^{-1}$ for all sufficiently large $t$.

It is not clear to us whether the bound $\left\|H S_{t}\right\| \leq c t^{-1}$ (for large $t$ ) could be derived from a weaker version $\left\|\left(I-S_{1}\right) S_{t}\right\| \leq c t^{-1}, t \geq 1$, of (8.2). If this were possible then we would only need Theorem 1.2, and not Theorem 1.13, to prove Theorem 1.14.

Proof of Theorem 1.15. Theorem 1.10 applied to the density $K=K_{1}$ gives

$$
K_{t}(g)+t^{1 / 2}\left|\left(\partial_{h} K_{t}\right)(g)\right| \leq c t^{-D / 2} e^{-b \rho(g)^{2} / t}
$$

for all $g \in G, h \in U$ and $t \in \mathbb{N}=\{1,2,3, \ldots\}$. But by the convolution identities $K_{t_{1}+t_{2}}=K_{t_{1}} * K_{t_{2}}, \partial_{h} K_{t_{1}+t_{2}}=\left(\partial_{h} K_{t_{1}}\right) * K_{t_{2}}$ and by (8.1), the bound (8.3) easily extends to all real $t \geq 1$.

Next, applying Theorem 1.14 and (8.3) we have

$$
\begin{aligned}
\left\|(d / d t)^{2} K_{t}\right\|_{\infty} & =\left\|H^{2} K_{t}\right\|_{\infty}=\left\|\left(H K_{t / 2}\right) *\left(H K_{t / 2}\right)\right\|_{\infty} \leq\left\|H K_{t / 2}\right\|_{2}^{2} \\
& \leq\left\|H S_{t / 4}\right\|_{2 \rightarrow 2}^{2}\left\|K_{t / 4}\right\|_{2}^{2} \leq c t^{-2} t^{-D / 2}
\end{aligned}
$$

for all $t \geq 4$. A Taylor expansion of the function $t \mapsto K_{t}(g)$ shows that

$$
\left|(d / d t) K_{t}(g)\right| \leq s^{-1}\left|K_{t+s}(g)\right|+s^{-1}\left|K_{t}(g)\right|+2^{-1} s \sup _{u \in[t, t+s]}\left|(d / d u)^{2} K_{u}(g)\right|
$$

for all $t, s>0$ and $g \in G$. By choosing $s=t e^{-\varepsilon \rho(g)^{2} / t}$ for a sufficiently small constant $\varepsilon>0$, and applying (8.3), (8.4), we deduce that

$$
\left|(d / d t) K_{t}(g)\right| \leq c^{\prime} t^{-1} t^{-D / 2} e^{-b^{\prime} \rho(g)^{2} / t} \quad \text { for } t \geq 4 .
$$

Finally, let $X$ be a right invariant vector field on $G$. A standard local regularity estimate for subelliptic operators (cf. [38, Corollary III.1.3]) implies that

$$
|X F(0, e)| \leq c \sup _{(s, h) \in(-1,1) \times U}|F(s, h)|
$$

for all solutions $F:(-1,1) \times U \rightarrow \mathbb{R}$ of the heat equation $(\partial /(\partial t)+H) F=0$. Applying this to the functions $F^{(t, g)}$ defined by

$$
F^{(t, g)}(s, h):=K_{t+s}(h g)-K_{t}(g)
$$

gives

$$
\left|X K_{t}(g)\right| \leq c \sup _{(s, h) \in(-1,1) \times U}\left|K_{t+s}(h g)-K_{t}(g)\right|
$$

for all $t \geq 2$ and $g \in G$. The desired Gaussian bound on $X K_{t}(g)$ then follows easily from (8.3) and (8.5). 


\section{Locally compact groups}

In this section and the next, our aim is to prove the results of Subsection 1.4. In general, $G$ will denote a compactly generated locally compact group, and $G_{0}=\overline{[G, G]}, G_{1}$ are the closed normal subgroups of $G$ defined as in Subsection 1.4.

We fix some notation. For any locally compact group $H$, the connected component containing the identity of $H$ will be denoted $H^{c}$. Note that $H^{c}$ is always a closed normal subgroup of $H$ and the quotient $H / H^{c}$ is a totally disconnected locally compact group (cf. [23, Chapter II]); $H / H^{c}$ is discrete if and only if $H^{c}$ is open in $H$, which is not always the case. Recall that $H$ is said to be almost connected if $H / H^{c}$ is compact.

Lie groups are not assumed to be connected in what follows. If $H$ is a Lie group, however, then $H^{c}$ is an open subgroup of $H$, and $H / H^{c}$ is a countable discrete group.

We shall need the following fundamental structure theorems for locally compact groups, for which [27] is a standard reference.

Theorem 9.1 ([27, Section 4.6]) Let $H$ be an almost connected locally compact group. Then there exists a compact normal subgroup $N$ of $H$ such that $H / N$ is a Lie group.

Theorem 9.2 ([27, Section 2.3]) Let $H$ be a locally compact group. There exists an open subgroup $H^{\prime}$ of $H$ such that $H^{\prime}$ is almost connected.

By a one-parameter subgroup of a locally compact group $H$ we mean a continuous homomorphism $\theta: \mathbb{R} \rightarrow H$.

Theorem 9.3 ([27, Section 4.15]) Let $H$ be a locally compact group, $N$ a compact normal subgroup of $H$, and $p: H \rightarrow H / N$ the canonical homomorphism. If $\tilde{\theta}$ is a one-parameter subgroup of $H / N$, then there exists a one-parameter subgroup $\theta$ of $H$ such that $\tilde{\theta}(t)=p(\theta(t))$ for all $t \in \mathbb{R}$.

Proof of Theorem 1.16. Let $K$ be centered and satisfy Assumption (i)'. Just as in the proof of Lemma 2.5 (see the Remark following Lemma 2.4) we have an estimate

$$
\operatorname{Re}((I-T) f, f) \geq c \Gamma_{2}(f)^{2}
$$

for all $f \in L^{2}$. Then analyticity of $T$ in $L^{2}$ is a consequence of the arguments of Section 2 together with (1.9). Analyticity in $L^{p}(1<p<\infty)$ then follows as in Corollary 1.4.

Therefore, it only remains to prove (1.9). For this we need the following version of (3.1). 
Proposition 9.4 Let $C$ be any compact subset of $G_{1}$. Then there exists $c=c(C)>0$ such that

$$
\left|\left(\partial_{g} f_{1}, f_{2}\right)\right| \leq c \Gamma_{2}\left(f_{1}\right) \Gamma_{2}\left(f_{2}\right)
$$

for all $g \in C$ and $f_{1}, f_{2} \in L^{2}$.

The proof of Proposition 9.4 is rather technical and is deferred to Section 10 .

Next, consider the homomorphism $\pi_{1}: G \rightarrow G / G_{1} \cong \mathbb{R}^{q} \times \mathbb{Z}^{r}$, and identify $G / G_{1}=\mathbb{R}^{q} \times \mathbb{Z}^{r}$. Let $e_{1}, \ldots, e_{q+r} \in \mathbb{Z}^{q+r} \subseteq \mathbb{R}^{q} \times \mathbb{Z}^{r}$ be the standard basis vectors, that is, $e_{j}$ has a 1 in the $j$-th position and zeroes elswhere.

As in the proof of Theorem 1.3 for Lie groups, we wish to find oneparameter subgroups with the following property.

Lemma 9.5 There exist one-parameter subgroups $x_{1}, \ldots, x_{q}$ of $G$ such that $\pi_{1}\left(x_{j}(t)\right)=t e_{j}$ for all $t \in \mathbb{R}$ and $j \in\{1, \ldots, q\}$.

Proof. By general topological group theory, $\pi_{1}\left(G^{c}\right)$ is a dense, connected subgroup of the group $\left(G / G_{1}\right)^{c}=\mathbb{R}^{q} \times\{0\}$ (see [23, Theorem II.7.12]). Applying Theorem 9.1 to $G^{c}$, we choose a compact normal subgroup $N$ of $G^{c}$ such that $H^{\prime}:=G^{c} / N$ is a Lie group. Note that $N \subseteq G_{1}$ by compactness of $N$. Therefore, the homomorphism $\left.\pi_{1}\right|_{G^{c}}: G^{c} \rightarrow \mathbb{R}^{q} \times\{0\}$ induces a homomorphism $\pi_{2}: H^{\prime} \rightarrow \mathbb{R}^{q} \times\{0\}$ with $\pi_{2}(g N)=\pi_{1}(g), g \in G^{c}$. Thus $\pi_{1}\left(G^{c}\right)=\pi_{2}\left(H^{\prime}\right)$ is a dense, connected Lie subgroup of $\mathbb{R}^{q} \times\{0\}$, and hence $\pi_{1}\left(G^{c}\right)=\pi_{2}\left(H^{\prime}\right)=\mathbb{R}^{q} \times\{0\}$.

Since $H^{\prime}$ is a Lie group, as in the proof of Theorem 1.3 one can find one-parameter subgroups $y_{1}, \ldots, y_{q}$ of $H^{\prime}$ such that $\pi_{2}\left(y_{j}(t)\right)=t e_{j}, j \in$ $\{1, \ldots, q\}$. Applying Theorem 9.3 to $G^{c}$ yields the existence of one-parameter subgroups $x_{1}, \ldots, x_{q}$ of $G^{c}$ such that $y_{j}(t)=x_{j}(t) N$ for all $t \in \mathbb{R}$. These subgroups satisfy the lemma.

Next, fix elements $z_{1}, \ldots, z_{r} \in G$ such that $\pi_{1}\left(z_{j}\right)=e_{q+j}$ for $j \in$ $\{1, \ldots, r\}$. Given any $g \in G$, we set $t_{j}=\pi_{1}^{(j)}(g), j \in\{1, \ldots, q+r\}$, and write

$$
g=x_{1}\left(t_{1}\right) \cdots x_{q}\left(t_{q}\right) z_{1}^{t_{q+1}} \cdots z_{r}^{t_{q+r}} g^{\prime}
$$

where $g^{\prime} \in G$. Then $g^{\prime} \in G_{1}$, because

$$
\pi_{1}(g)=\left(t_{1}, \ldots, t_{q+r}\right)=\pi_{1}\left(x_{1}\left(t_{1}\right) \cdots x_{q}\left(t_{q}\right) z_{1}^{t_{q+1}} \cdots z_{r}^{t_{q+r}}\right) .
$$

Let $C \subseteq G$ be any compact set. By applying identity (2.4) and arguing as in the proof of Theorem 1.3, one finds that

$$
\partial_{g}=\sum_{j=1}^{q} \pi_{1}^{(j)}(g) \partial_{x_{j}(1)}+\sum_{k=1}^{r} \pi_{1}^{(q+k)}(g) \partial_{z_{k}}+V_{g}
$$


where $V_{g}$ satisfies an estimate

$$
\left|\left(V_{g} f_{1}, f_{2}\right)\right| \leq c(C) \Gamma_{2}\left(f_{1}\right) \Gamma_{2}\left(f_{2}\right)
$$

for all $f_{1}, f_{2} \in L^{2}$ and all $g \in C$, with $c(C)>0$ a constant depending on $C$. The proof of (9.1) follows the argument of Theorem 1.3 with the following main differences: first, one now applies Proposition 9.4 to bound the term $\left(\partial_{g^{\prime}} f_{1}, f_{2}\right)$, and secondly, one requires estimates of form

$$
\left|\left(\left(\partial_{z_{j}^{m}}-m \partial_{z_{j}}\right) f_{1}, f_{2}\right)\right| \leq c(m) \Gamma_{2}\left(f_{1}\right) \Gamma_{2}\left(f_{2}\right)
$$

for all $j \in\{1, \ldots, r\}, m \in \mathbb{Z}$ and $f_{1}, f_{2} \in L^{2}$. The latter estimates are easily obtained, since using (2.4) and the identity $\partial_{z_{j}^{-1}}=-\partial_{z_{j}}-\partial_{z_{j}} \partial_{z_{j}^{-1}}$ one may express $\partial_{z_{j}^{m}}-m \partial_{z_{j}}$ as a finite linear combination of terms each of form

$$
\partial_{z_{j}} \varepsilon_{1} \ldots \partial_{z_{j}}^{\varepsilon_{l}}
$$

where $l \geq 2$ and $\varepsilon_{1}, \ldots \varepsilon_{l} \in\{-1,+1\}$.

Finally, since $K$ is centered, by choosing $C$ to contain the compact support of $K$ then (9.1) yields

$$
\left|\left((I-T) f_{1}, f_{2}\right)\right|=\left|\int_{G} d g K(g)\left(V(g) f_{1}, f_{2}\right)\right| \leq c \Gamma_{2}\left(f_{1}\right) \Gamma_{2}\left(f_{2}\right),
$$

proving (1.9).

This ends the proof of Theorem 1.16, modulo Proposition 9.4.

Proof of Theorem 1.17. It is enough to prove Statement (I) of the theorem, that is, the estimate (1.10). For then Statements (II) and (III) can be deduced just as in the proofs of Theorems 1.5, 1.6 and 1.8.

To obtain (1.10), we need the following variation of Proposition 9.4.

Proposition 9.6 Let $C \subseteq G_{1}$ be compact. Then there exists $c=c(C)>0$ such that

$$
\left|\left(\partial_{g} f_{1}, f_{2}\right)\right| \leq c \Gamma_{\infty}\left(f_{1}\right) \Gamma_{1}\left(f_{2}\right)
$$

for all $g \in C$ and $f_{1} \in L^{\infty}, f_{2} \in L^{1}$.

Reasoning as in the proof of Theorem 1.16 then shows, analogously to (9.1), that given a compact $C \subseteq G$ one has

$$
\left|\left(V_{g} f_{1}, f_{2}\right)\right| \leq c(C) \Gamma_{\infty}\left(f_{1}\right) \Gamma_{1}\left(f_{2}\right)
$$

for all $g \in C, f_{1} \in L^{\infty}, f_{2} \in L^{1}$. Then (1.10) follows using the hypothesis that $K$ is centered. Theorem 1.17 is proved. 
Proof of Theorem 1.18. This follows the proof of Theorem 1.10.

Proof of Theorem 1.19. The proof of Theorem 1.19 follows that of Theorem 1.7. The only significant difference occurs when proving that Conditions (II) or (IV) fail for non-centered $K$; one must also consider the case where $P$ (the image of $K$ under $\pi_{1}^{(j)}$ ) is a non-centered density on $\mathbb{Z}$. For such $P$, the relevant analysis is already given in, for example, [16].

Proof of Theorem 1.20. The proof of Theorem 1.17 goes through uniformly for the densities $\left(K_{n}\right)_{n=1}^{\infty}$, and the desired estimates follow just as in the proof of Theorem 1.11.

\section{Proof of Propositions 9.4 and 9.6}

Let $G$ be locally compact and compactly generated. In this section, we will prove Proposition 9.4; the proof of Proposition 9.6 requires only obvious changes and is omitted.

The proof of Proposition 9.4 reduces to the case where $G$ is second countable, by the following theorem and lemma.

Theorem 10.1 ([23, Theorem II.8.7]) Let $H$ be a locally compact group which is $\sigma$-compact (that is, $H$ is the union of some countable family of compact subsets of $H$; in particular, any compactly generated group is $\sigma$ compact). Then there exists a compact normal subgroup $N$ of $H$ such that $H / N$ is a second countable locally compact group.

Lemma 10.2 Let $N$ be a compact normal subgroup of $G$. If Proposition 9.4 holds with respect to the group $G^{\prime}:=G / N$, then it also holds with respect to $G$.

The proof of Lemma 10.2 is not difficult and is left to the reader.

Thus, in the rest of this section we shall assume that $G$ is second countable.

The next step in the proof is to obtain the desired estimate in case $g \in G_{0}$, as follows.

Proposition 10.3 For each $g \in G_{0}$, there exists a $c(g)>0$ such that

$$
\left|\left(\partial_{g} f_{1}, f_{2}\right)\right| \leq c(g) \Gamma_{2}\left(f_{1}\right) \Gamma_{2}\left(f_{2}\right)
$$

for all $f_{1}, f_{2} \in L^{2}$. 
The final step consists in extending the estimate from $G_{0}$ to $G_{1}$; this extension depends on the compactness of $G_{1} / G_{0}$.

We begin the proof of Proposition 10.3 with the following observation.

Lemma 10.4 Let $N$ be any compact subgroup of $G$. Then an estimate (10.1) holds for each $g \in[G, G] N=N[G, G]$.

Proof. This is an straightforward adaption of the proofs of Lemmas 3.3 and 3.4, using (2.3), (2.5) and (2.6).

Proposition 10.3 would now follow if we had $G_{0} \subseteq N[G, G]$ with $N$ some compact subgroup of $G$. Unfortunately we do not know if such an $N$ exists in general, but we have the following substitute result. If $A_{1}, \ldots, A_{s}$ are subsets of a group $H$, denote by $\left\langle A_{1}, \ldots, A_{s}\right\rangle$ the subgroup of $H$ generated by $A_{1} \cup A_{2} \cup \cdots \cup A_{s}$.

Proposition 10.5 There exist a compact subgroup $N$ of $G_{0}$, and one-parameter subgroups $\theta_{1}, \ldots, \theta_{s}$ of $G_{0}\left(s \in \mathbb{N}_{0}=\{0,1,2, \ldots\}\right)$ such that $\theta_{j}(1) \in$ $[G, G] N$ for all $j$ and

$$
G_{0}=[G, G] N\left\langle\theta_{1}(\mathbb{R}), \ldots \theta_{s}(\mathbb{R})\right\rangle
$$

Proposition 10.5 is just a particular case of the following lemma (apply the lemma with $H=G_{0}=\overline{[G, G]}$ and $A=[G, G]$ ).

Lemma 10.6 Let $A$ be a dense subset of a locally compact group $H$. Then there exist a compact subgroup $N$ of $H$, and one-parameter subgroups $\theta_{1}, \ldots, \theta_{s}$ of $H\left(s \in \mathbb{N}_{0}\right)$ such that

$$
\theta_{j}(1) \in A N
$$

for all $j$, the set

$$
H^{\prime}:=N\left\langle\theta_{1}(\mathbb{R}), \ldots \theta_{s}(\mathbb{R})\right\rangle
$$

is an open subgroup of $H$ with $N$ a normal subgroup of $H^{\prime}$, and

$$
H=A H^{\prime}=A N\left\langle\theta_{1}(\mathbb{R}), \ldots, \theta_{s}(\mathbb{R})\right\rangle .
$$

Proof of Lemma 10.6. Equation (10.5) will follow from (10.4), because $H=\bar{A}=A V$ holds for any neighborhood $V$ of $e$ in $H$, hence for $V=H^{\prime}$ since $H^{\prime}$ is an open subgroup of $H$.

To prove the remaining statements of the lemma, we begin with some special cases of $H$. First, suppose that $H$ is a Lie group (not necessarily 
connected) with Lie algebra $\mathfrak{h}$. The exponential map exp $: \mathfrak{h} \rightarrow H$ maps some open neighborhood of $0 \in \mathfrak{h}$ diffeomorphically onto a open neighborhood $W$ of $e$ in $H$. Since $A \cap W$ is dense in $W$, it is easy to deduce the existence of a vector space basis $X_{1}, \ldots, X_{s}$ of $\mathfrak{h}$ such that $\exp \left(X_{j}\right) \in A \cap W$ for all $j$. Setting $\theta_{j}(t)=\exp \left(t X_{j}\right), t \in \mathbb{R}$, we have $\theta_{j}(1) \in A$ and $H^{c}=$ $\left\langle\theta_{1}(\mathbb{R}), \ldots, \theta_{s}(\mathbb{R})\right\rangle$. Now $H^{c}$ is open in $H$ since $H$ is a Lie group, so (10.3) and (10.4) hold with $N=\{e\}$ and $H^{\prime}=H^{c}$.

Next, suppose that $H$ is an almost connected locally compact group. By Theorem 9.1, choose a compact normal subgroup $N$ of $H$ such that $\widetilde{H}:=H / N$ is a Lie group, and let $p: H \rightarrow \widetilde{H}$ be the canonical map.

Since $p(A)$ is dense in $\widetilde{H}$, by applying the preceding case we find oneparameter subgroups $\widetilde{\theta}_{1}, \ldots, \widetilde{\theta}_{s}$ of $\widetilde{H}$ such that $\widetilde{\theta}_{j}(1) \in p(A)$ and

$$
\widetilde{H}^{c}=\left\langle\widetilde{\theta}_{1}(\mathbb{R}), \ldots, \widetilde{\theta}_{s}(\mathbb{R})\right\rangle
$$

By Theorem 9.3 there exist one-parameter subgroups $\theta_{1}, \ldots, \theta_{s}$ of $H$ such that $\widetilde{\theta}_{j}(t)=p\left(\theta_{j}(t)\right), t \in \mathbb{R}$. Then $\theta_{j}(1) \in p^{-1}(p(A))=A N$ for all $j$. Setting $H^{\prime}:=p^{-1}\left(\widetilde{H}^{c}\right)$, we observe that $H^{\prime}$ is an open subgroup of $H$ and that $H^{\prime}=N\left\langle\theta_{1}(\mathbb{R}), \ldots, \theta_{s}(\mathbb{R})\right\rangle$. This proves the lemma for $H$ almost connected.

Finally, for $H$ any locally compact group we choose by Theorem 9.2 an open, almost connected subgroup $\widehat{H}$ of $H$. Since $\widehat{A}:=A \cap \widehat{H}$ is dense in $\widehat{H}$, we may apply the preceding case to $\widehat{H}$ to reach the desired conclusion.

Proof of Proposition 10.3. Choose $N$ and $\theta_{1}, \ldots, \theta_{s}$ as in Proposition 10.5. By Lemma 10.4, the desired estimate (10.1) holds when $g=\theta_{j}(1)$ for some $j$. One proves in the same way as (3.9) that, for each $t \in \mathbb{R}$, there exists $c(t)>0$ with

$$
\left|\left(\left(\partial_{\theta_{j}(t)}-t \partial_{\theta_{j}(1)}\right) f_{1}, f_{2}\right)\right| \leq c(t) \Gamma_{2}\left(f_{1}\right) \Gamma_{2}\left(f_{2}\right)
$$

for all $f_{1}, f_{2} \in L^{2}$. Therefore, (10.1) holds whenever $g \in \theta_{j}(\mathbb{R})$. Then from (10.2), from Lemma 10.4 and by applying (2.3), (2.5), (2.6), it is straightforward to deduce (10.1) for each $g \in G_{0}$.

We next show that the estimate of Proposition 10.3 self-improves into a uniform estimate over compacta.

Lemma 10.7 Let $C$ be any compact subset of $G_{0}$. Then there exists $c=$ $c(C)>0$ such that

$$
\left|\left(\partial_{g} f_{1}, f_{2}\right)\right| \leq c \Gamma_{2}\left(f_{1}\right) \Gamma_{2}\left(f_{2}\right)
$$

for all $g \in C$ and $f_{1}, f_{2} \in L^{2}$. 
Proof. For each $m \in \mathbb{N}$, let $C_{m}$ be the set of all $g \in G_{0}$ for which the estimate $\left|\left(\partial_{g} f_{1}, f_{2}\right)\right| \leq m \Gamma_{2}\left(f_{1}\right) \Gamma_{2}\left(f_{2}\right)$ holds for all $f_{1}, f_{2} \in L^{2}$. By taking limits in this estimate, it is clear that $C_{m}$ is closed in $G_{0}$. Moreover, $G_{0}=\bigcup_{m=1}^{\infty} C_{m}$ by Proposition 10.3. The Baire theorem for locally compact Hausdorff spaces (see [23, Appendix B.19]), applied to $G_{0}$, implies that $C_{m_{0}}$ has non-empty interior for some $m_{0} \in \mathbb{N}$.

Select therefore a non-empty open subset $A$ of $G_{0}$ with compact closure $\bar{A}$, such that $\bar{A} \subseteq C_{m_{0}}$. Given a compact set $C \subseteq G_{0}$, we may find points $h_{1}, \ldots, h_{k} \in G_{0}$ such that $C \subseteq \bigcup_{i=1}^{k} h_{i} A$. For $g \in C$ write $g=h_{i} a$ where $a \in A$, and note that $\sup \{\rho(b): b \in A\}<\infty$ by compactness of $\bar{A}$. Then by Proposition 10.3, (2.3), (2.5) and (2.6) we obtain

$$
\left|\left(\partial_{g} f_{1}, f_{2}\right)\right| \leq\left|\left(\partial_{h_{i}} f_{1}, f_{2}\right)\right|+\left|\left(\partial_{a} f_{1}, L\left(h_{i}^{-1}\right) f_{2}\right)\right| \leq c \Gamma_{2}\left(f_{1}\right) \Gamma_{2}\left(f_{2}\right)
$$

where $c$ is independent of $g \in C$.

To continue the proof of Proposition 9.4, we exploit the compactness of the quotient group $G_{1} / G_{0} \cong M$. Let us identify $M=G_{1} / G_{0}$. Since $G_{0}$ is a closed subgroup of the second countable, locally compact group $G_{1}$, by a standard result there exists a Borel set $\Sigma \subseteq G_{1}$ which meets each coset $g G_{0}$ ( $g \in G_{1}$ ) in exactly one point; since $M$ is compact, we may choose $\Sigma$ to have compact closure in $G_{1}$ (see [25, Lemma 1.1]).

If we denote by $\pi: G_{1} \rightarrow G_{1} / G_{0}=M$ the canonical projection, then $\left.\pi\right|_{\Sigma}: \Sigma \rightarrow M$ is a measurable bijection. Let $q: M \rightarrow \Sigma \subseteq G$ be the inverse of $\left.\pi\right|_{\Sigma}$, so that $\pi(q(a))=a, a \in M$. Consider the Haar measure $d a$ on $M$, normalized so $d a(M)=1$, and let $d y$ be the image measure of $d a$ under $q: M \rightarrow \Sigma$. Then $d y(\Sigma)=1$, and

$$
\int_{\Sigma} d y f(y)=\int_{M} d a(f \circ q)(a)
$$

for all $f \in L^{1}(\Sigma ; d y)$. The following result is essentially a generalization of the identity (3.2).

Lemma 10.8 Let $g \in G_{1}$, and for each $y \in \Sigma$ define $z^{y}=z(g, y) \in G_{1}$ by

$$
z^{y}:=q\left(\pi\left(y g^{-1}\right)\right)\left(y g^{-1}\right)^{-1}
$$

Then $z^{y} \in G_{0}$, and for each $f \in L^{p}=L^{p}(G ; d g)$ one has

$$
\partial_{g} f=-\int_{\Sigma} d y \partial_{g} \partial_{y^{-1}} f+\int_{\Sigma} d y L\left(q\left(\pi\left(y g^{-1}\right)\right)^{-1}\right) \partial_{z^{y}} f
$$


Proof. The definition of $z^{y}$ implies that $\pi\left(z^{y}\right)$ is the identity element of $M=G_{1} / G_{0}$, so that $z^{y} \in G_{0}$.

It suffices to prove that the identity for $\partial_{g} f$ holds pointwise for any continuous function $f: G \rightarrow \mathbb{C}$. We start by observing that

$$
\begin{gathered}
\left(\partial_{g} f\right)(e)=f\left(g^{-1}\right)-f(e) \\
=f\left(g^{-1}\right)-\int_{\Sigma} d y f\left(y g^{-1}\right)-f(e)+\int_{\Sigma} d y f(y)+\int_{\Sigma} d y f\left(y g^{-1}\right)-\int_{\Sigma} d y f(y) \\
=-\int_{\Sigma} d y\left(\partial_{g} \partial_{y^{-1}} f\right)(e)+\int_{\Sigma} d y\left[f\left(y g^{-1}\right)-f\left(q\left(\pi\left(y g^{-1}\right)\right)\right)\right] \\
\quad+\int_{\Sigma} d y f\left(q\left(\pi\left(y g^{-1}\right)\right)\right)-\int_{\Sigma} d y f(y)
\end{gathered}
$$

where we used that $d y(\Sigma)=1$. The last two integrals in the right side cancel each other; indeed, with $F:=f \circ q$ we have from (10.6) that $\int_{\Sigma} d y f(y)=$ $\int_{M} d a F(a)$ and

$$
\int_{\Sigma} d y f\left(q\left(\pi\left(y g^{-1}\right)\right)\right)=\int_{M} d a F\left(a \pi\left(g^{-1}\right)\right)=\int_{M} d a F(a) .
$$

Because

$$
\int_{\Sigma} d y\left[f\left(y g^{-1}\right)-f\left(q\left(\pi\left(y g^{-1}\right)\right)\right)\right]=\left(\int_{\Sigma} d y L\left(q\left(\pi\left(y g^{-1}\right)\right)^{-1}\right) \partial_{z^{y}} f\right)(e),
$$

we obtain the desired identity at the point $e \in G$. The identity at any point $h \in G$ then follows by replacing $f$ with its right translate $R_{G}(h) f$ where $\left(R_{G}(h) f\right)(k)=f(k h), k \in G$.

Now suppose that $C \subseteq G_{1}$ is compact and $g \in C$. By Lemma 10.8,

$$
\left|\left(\partial_{g} f_{1}, f_{2}\right)\right| \leq \int_{\Sigma} d y\left|\left(\partial_{y^{-1}} f_{1}, \partial_{g^{-1}} f_{2}\right)\right|+\int_{\Sigma} d y\left|\left(\partial_{z^{y}} f_{1}, L\left(q\left(\pi\left(y g^{-1}\right)\right)\right) f_{2}\right)\right|
$$

for $f_{1}, f_{2} \in L^{2}$. Then Proposition 9.4 follows by applying (2.5) to estimate the first integral over $\Sigma$, and applying Lemma 10.7 and (2.6) to estimate the second integral since $z^{y} \in G_{0}$. Here we must observe that, because $\Sigma$ has compact closure and $y, q\left(\pi\left(y g^{-1}\right)\right) \in \Sigma$, the elements $z^{y}=z(g, y)$ remain within a fixed compact subset of $G_{0}$ as $g$ varies over $C$. The proof of Proposition 9.4 is complete.

Acknowledgements. This work was carried out with financial support from the Australian Research Council (ARC) Centre of Excellence for Mathematics and Statistics of Complex Systems (MASCOS).

I thank George Willis for valuable advice on the structure theory of locally compact groups. 


\section{References}

[1] Alexopoulos, G.: Sous-laplaciens et densités centrés sur les groupes de Lie à croissance polynomiale du volume. C. R. Acad. Sci. Paris Sér. I Math. 326 (1998), 539-542.

[2] Alexopoulos, G.: Sub-Laplacians with drift on Lie groups of polynomial volume growth. Mem. Amer. Math. Soc. 155 (2002), no. 739.

[3] Alexopoulos, G.: Random walks on discrete groups of polynomial volume growth. Ann. Probab. 30 (2002), 723-801.

[4] Alexopoulos, G.: Centered densities on Lie groups of polynomial volume growth. Probab. Theory Relat. Fields 124 (2002), 112-150.

[5] Blunck, S.: Perturbation of analytic operators and temporal regularity of discrete heat kernels. Colloq. Math. 86 (2000), 189-201.

[6] Blunck, S.: Analyticity and discrete maximal regularity on $L_{p}$-spaces. J. Funct. Anal. 183 (2001), 211-230.

[7] Bounechada, N.: Distorsion des distances dans les groupes de Lie nilpotents. Bull. Sci. Math. 127 (2003), 797-813.

[8] Carne, T.K.: A transmutation formula for Markov chains. Bull. Sci. Math. (2) 109 (1985), 399-405.

[9] Coulhon, T., Grigor'yan, A. And ZuccA, F.: The discrete integral maximum principle and its applications. Tohoku Math. J. (2) 57 (2005), $559-587$.

[10] Coulhon, T. And Saloff-Coste, L.: Puissances d'un opérateur régularisant. Ann. Inst. H. Poincaré Probab. Statist. 26 (1990), 419-436.

[11] Colfman, R. R. And Weiss, G.: Transference methods in analysis. CBMS Regional Conference Series in Mathematics 31. American Mathematical Society, Providence, 1976.

[12] Davies, E. B.: Heat kernels and spectral theory. Cambridge Tracts in Mathematics 92. Cambridge University Press, Cambridge, 1989.

[13] Davies, E. B.: Uniformly elliptic operators with measurable coefficients. J. Funct. Anal. 132 (1995), 141-169.

[14] Dungey, N.: On Gaussian kernel estimates on groups. Colloq. Math. 100 (2004), 77-90.

[15] Dungey, N.: Heat kernel and semigroup estimates for sublaplacians with drift on Lie groups. Publ. Mat. 49 (2005), 375-391.

[16] Dungey, N.: Properties of random walks on discrete groups: time regularity and off-diagonal estimates. To appear in Bull. Sci. Math.

[17] Dungey, N.: A note on time regularity for discrete time heat kernels. Semigroup Forum 72 (2006), no. 3, 404-410.

[18] Grigor'yan, A.: Estimates of heat kernels on Riemannian manifolds. In Spectral theory and geometry (Edinburgh, 1998), 140-225. London Math. Soc. Lecture Note Ser. 273. Cambridge Univ. Press, Cambridge, 1999. 
[19] Grigorchuk, R.: Degrees of growth of finitely generated groups and the theory of invariant means. Izv. Akad. Nauk. SSSR Ser. Mat. 48 (1984), no. 5, 939-985.

[20] Guivarc'H, Y.: Croissance polynomiale et périodes des fonctions harmoniques. Bull. Soc. Math. France 101 (1973), 333-379.

[21] Hebisch, W.: On heat kernels on Lie groups. Math. Z. 210 (1992), no. 4, 593-605.

[22] Hebisch, W. and Saloff-Coste, L.: Gaussian estimates for Markov chains and random walks on groups. Ann. Probab. 21 (1993), 673-709.

[23] Hewitt, E. And Ross, K. A.: Abstract Harmonic Analysis. I. Structure of topological groups, integration theory, group representations. Fundamental Principles of Mathematical Sciences 115. Springer-Verlag, Berlin-New York, 1979.

[24] Kato, T.: Perturbation theory for linear operators, Grundlehren der Mathematischen Wissenschaften 132. Springer-Verlag, Berlin, 1976.

[25] Mackey, G.: Induced representations of locally compact groups I. Ann. of Math. (2) $\mathbf{5 5}$ (1952), 101-139.

[26] Melzi, C.: Large time estimates for heat kernels in nilpotent Lie groups. Bull. Sci. Math. 126 (2002), 71-86.

[27] Montgomery, D. And Zippin, L.: Topological transformation groups. Interscience Publishers, New York-London, 1955.

[28] Mustapha, S.: Gaussian estimates for heat kernels on Lie groups. Math. Proc. Cambridge Philos. Soc. 128 (2000), 45-64.

[29] Nevanlinna, O.: Convergence of iterations for linear equations. Lectures in Mathematics ETH Zürich. Birkhäuser, Basel, 1993.

[30] Nevanlinna, O.: On the growth of the resolvent operators for power bounded operators. In Linear Operators (Warsaw, 1994), 247-264. Banach Center Publ. 38. Polish Acad. Sci., Warsaw, 1997,

[31] Paterson, A.: Amenability. Mathematical Surveys and Monographs 29. American Mathematical Society, Providence, 1988.

[32] Robinson, D. W.: Elliptic operators and Lie groups. Oxford Mathematical Monographs. Oxford University Press, New York, 1991

[33] Varadarajan, V.S.: Lie groups, Lie algebras, and their representations, Graduate Texts in Mathematics 102. Springer-Verlag, New York, 1984.

[34] Varopoulos, N. T.: Long range estimates for Markov chains. Bull. Sci. Math. (2) 109 (1985), 225-252.

[35] Varopoulos, N. T.: Analysis on Lie groups. Rev. Mat. Iberoamericana 12 (1996), 791-917.

[36] Varopoulos, N. T.: Distance distortion on Lie groups. In: Random walks and discrete potential theory (Cortona, 1997), 320-357. Sympos. Math. XXXIX. Cambridge Univ. Press, Cambridge, 1999. 
634 N. Dungey

[37] Varopoulos, N. T.: Geometric and potential theoretic results on Lie groups. Canad. J. Math. 52 (2000), 412-437.

[38] Varopoulos, N. T., Saloff-Coste, L. and Coulhon, T.: Analysis and geometry on groups. Cambridge Tracts in Mathematics 100. Cambridge University Press, Cambridge, 1992.

Recibido: 6 de septiembre de 2005

Nick Dungey

Department of Mathematics

Macquarie University

NSW 2109, Australia.

ndungey@ics.mq.edu.au 